\title{
Extracellular vesicles: biomarkers and regulators of vascular function during extracorporeal circulation
}

\author{
Mark J. McVey ${ }^{1,2,3,4}$ and Wolfgang M. Kuebler ${ }^{1,2,5,6,7}$ \\ ${ }^{1}$ Keenan Research Centre for Biomedical Science, St Michael's Hospital, Toronto, ON, Canada \\ ${ }^{2}$ Department of Physiology, University of Toronto, Toronto, ON, Canada \\ ${ }^{3}$ Department of Anesthesia, University of Toronto, Toronto, ON, Canada \\ ${ }^{4}$ Department of Anesthesia and Pain Medicine, SickKids, Toronto, ON, Canada \\ ${ }^{5}$ Department of Surgery, University of Toronto, Toronto, ON, Canada \\ ${ }^{6}$ Institute of Physiology, Charité-Universitätsmedizin Berlin, Berlin, Germany \\ ${ }^{7}$ German Heart Institute, Berlin, Germany \\ Correspondence to: Wolfgang M. Kuebler, email: wolfgang.kuebler@charite.de \\ Keywords: extracellular vesicles; extracorporeal circuits; ECMO; cardiopulmonary bypass; hemodialysis \\ Received: May 26, $2018 \quad$ Accepted: November 26, $2018 \quad$ Published: December 14, 2018 \\ Copyright: McVey et al. This is an open-access article distributed under the terms of the Creative Commons Attribution License \\ 3.0 (CC BY 3.0), which permits unrestricted use, distribution, and reproduction in any medium, provided the original author and \\ source are credited.
}

\section{ABSTRACT}

Extracellular vesicles (EVs) are generated at increased rates from parenchymal and circulating blood cells during exposure of the circulation to abnormal flow conditions and foreign materials associated with extracorporeal circuits (ExCors). This review describes types of EVs produced in different ExCors and extracorporeal life support (ECLS) systems including cardiopulmonary bypass circuits, extracorporeal membrane oxygenation (ECMO), extracorporeal carbon dioxide removal ( $\left.E \mathrm{CCO}_{2} \mathrm{R}\right)$, apheresis, dialysis and ventricular assist devices. Roles of EVs not only as biomarkers of adverse events during ExCor/ECLS use, but also as mediators of vascular dysfunction are explored. Manipulation of the number or subtypes of circulating EVs may prove a means of improving vascular function for individuals requiring ExCor/ECLS support. Strategies for therapeutic manipulation of EVs during ExCor/ECLS use are discussed such as accelerating their clearance, preventing their genesis or pharmacologic options to reduce or select which and how many EVs circulate. Strategies to reduce or select for specific types of EVs may prove beneficial in preventing or treating other EV-related diseases such as cancer.

\section{INTRODUCTION}

Use of extracorporeal circuits (ExCor) and extracorporeal life support (ECLS) in medicine has steadily increased over time. Early applications of ExCor/ ECLS include hemodialysis (HD), with initial accounts dating back to the nineteen twenties. Cardiopulmonary bypass $(\mathrm{CPB})$ and cardiac and respiratory extracorporeal life support (cECLS and rECLS respectively) systems emerged in the nineteen fifties to seventies. Since then, distinct specialized ECs have been developed to meet growing demands to support critically ill patients, including apheresis machines, ventricular assist devices
(VADs), extracorporeal membrane oxygenators (ECMO) and extracorporeal carbon dioxide removal $\left(\mathrm{ECCO}_{2} \mathrm{R}\right)$ devices such as the nova-lung and A-lung (Table 1). Examples of critically ill patients benefiting from ExCor and ECMO include both solid organ and hematological oncology patients $[1,2]$. Though typically utilized in hospitals, a growing trend towards home HD programs or enhanced portability of ExCor/ECLS such as VADs has allowed patients to leave hospital and increased patient mobility opening the possibility of long term destination therapy for conditions such as heart failure with use of prolonged-ECLS in the form of VADs [3, 4]. As such, the use of an increasingly complex range of ExCor/ECLS is 
expanding in terms of the number of patients utilizing this evolving resource both in and out of the hospital.

As ExCor/ECLS use rises within increasingly diverse patient populations (including oncology patients) there is a growing need to develop biomarkers capable of predicting ExCor/ECLS associated adverse events. Available biomarkers are currently limited at predicting ExCor/ECLS adverse events. Notably, ExCor/ECLS leads to generation of elevated blood levels of extracellular vesicles (EVs). EVs are emerging as potential biomarkers of disease. This review aims to characterize EVs associated with use of different ExCor/ECLS systems and discuss their potential roles as biomarkers and effectors of ExCor/ECLS associated adverse events. Lastly, the use of ExCor as a strategy for therapeutic manipulation of EVs will be discussed such as systems capable of reducing or selecting specific EVs.

\section{REVIEW METHODOLOGY}

Studies included in this narrative review examining available literature on EVs and extracorporeal support (ExCor/ECLS) were obtained by searching the PubMed electronic database during 2016-2018 using the following keywords: extracorporeal, ECMO, ECLS, hemodialysis, Novalung, cardiopulmonary bypass, cell saver, VAD, LVAD, BIVAD, RVAD, ventricle assist device, artificial heart, extracellular vesicles, microparticles, microvesicles, apoptotic bodies, exosomes. Searched review- and original-papers were excluded if they did not meet the following inclusion criteria: being published in English (or if translation to English was available) as well as having relevant titles and abstracts. The goal of this review is to introduce physicians (e.g. intensivists, oncologists) and scientists to the relevance and potential of EVs to influence patient care during ExCor/ECLS.

\section{INDICATIONS FOR EXCOR/ECLS USE}

Use of ExCor/ECLS has been revolutionary in supporting a wide range of previously fatal conditions such as end stage cardiopulmonary diseases and renal failure (Table 1) [5, 6]. ExCor/ECLS use can be shortterm for minutes to hours such as for cardiopulmonary bypass or longer such as days as seen with hemodialysis or VADs and finally long-term or destination use such as with prolonged-ECLS using VADs $[7,8]$. There is an ever-increasing number of different extracorporeal circuits entering clinical use (examples shown in Table 1) which corelates with increasing use of ExCor/ECLS with patients [9-13]. Though the interaction of ExCor/ECLS with the patients' physiological functions is intended to be supportive, due to their intimate connection with the patient, ExCor/ECLS can unfortunately lead to serious adverse events.

\section{EXCOR/ECLS ASSOCIATED ADVERSE EVENTS}

Compared with the human circulation (without ExCor/ECLS use), interaction with distinct ExCor/ ECLS systems create altered physiologic conditions for circulating cells including foreign surfaces, increased shear forces, perturbations in the flow of blood (pulsatile or continuous) and altered function of circulatory cells due to circuit components and use of medications related to ExCor/ECLS use such as anticoagulants (Tables 2-3). ExCor/ECLS adverse events are influenced in part by patient related factors such as pre-existing co-morbid disease states, medications being consumed, inflammation and circuit related factors such as the components within ExCor/ECLS systems (Tables 2-3). Specifically, within circuits, there are components which add risk for adverse events such as cannulas and tubing whose foreign material activates and reduces levels of circulating adhesive cells such as platelets or membrane oxygenators which through non-physiological shear forces traumatise RBCs leading to hemolysis. Specific components within ExCor/ECLS circuits such as oxygenators create local regions with high shear stresses $\left(8.4 \mathrm{~N} / \mathrm{m}^{2}\right)$ [14]. The consequences of blood flowing through such high shear environments depend in part on the magnitude of the shear rate, exposure time and the cells involved. Platelets exposed to high shear stresses for as little as 7 milliseconds (ms) release procoagulant phospholipids and after a $113 \mathrm{~ms}$ exposure platelets no longer respond to ADP agonist stimulation [15]. These physiologic changes can culminate in adverse events directly within the circulation and proximal organs (Table 2). With so many potential harms for patients it is important to monitor the proper function of ExCor/ ECLS systems and detect, predict, and - ideally - prevent or reduce adverse effects of ExCor/ECLS use which may lead to morbidity or even mortality.

\section{AVAILABLE BIOMARKERS FOR EXCOR/ECLS RELATED ADVERSE EVENTS}

Having an accurate means of monitoring detrimental effects is of crucial importance to maximize benefits while preventing adverse events related to ExCor/ECLS use. Though potentially useful, the available and emerging ExCor/ECLS biomarkers (Table 4) provide an incomplete picture of endothelial function, coagulopathy or degree of inflammation present and therefore highlight the need for further biomarker research.

\section{EXTRACELLULAR VESICLES}

EVs are rapidly emerging candidates to address the gap of reliable biomarkers for monitoring ExCor/ECLS 
Table 1: Extracorporeal circuit types describing specific indications, circuit functions, and components

\begin{tabular}{|c|c|c|c|}
\hline Circuit & Indication & Function & Components \\
\hline HD & $\begin{array}{l}\text { renal failure } \\
\text { acidosis } \\
\text { electrolyte abnormality } \\
\text { toxins } \\
\text { fluid overload } \\
\text { uremia }\end{array}$ & $\begin{array}{l}\text { removal of toxins } \\
\text { modification of circulating volume }\end{array}$ & $\begin{array}{l}\text { cannulas/needles } \\
\text { dialyser } \\
\text { pressure monitors } \\
\text { anticoagulant } \\
\text { pump } \\
\text { ultra-filtration unit } \\
\text { filters } \\
\text { heaters/heat exchangers } \\
\text { de-aerator } \\
\text { pH monitors }\end{array}$ \\
\hline CPB & $\begin{array}{l}\text { cardiac/pulmonary surgery } \\
\text { complex vascular surgery } \\
\text { airway surgery } \\
\text { brain surgery } \\
\text { deep hypothermic circulatory arrest } \\
\text { rewarming }\end{array}$ & $\begin{array}{l}\text { selective exclusion of cardiac and } \\
\text { vascular structures from circulation } \\
\text { oxygenation and } \mathrm{CO}_{2} \text { removal }\end{array}$ & $\begin{array}{l}\text { cannulas } \\
\text { oxygenator } \\
\text { heater } \\
\mathrm{CO}_{2} \text { exchanger } \\
\text { suction/vents } \\
\text { pump (roller/centrifugal) } \\
\text { blood storage chambers } \\
\text { hemo-concentrator } \\
\text { cardioplegia system } \\
\text { bubble detector/trap } \\
\text { anesthetic delivery unit/gas blender } \\
\text { blood reservoirs }\end{array}$ \\
\hline apheresis & $\begin{array}{l}\text { immune therapy } \\
\text { blood donation } \\
\text { neurologic diseases } \\
\text { hematologic diseases } \\
\text { metabolic diseases } \\
\text { dermatologic diseases } \\
\text { rheumatologic diseases } \\
\text { renal diseases } \\
\text { intoxications } \\
\text { neoplastic diseases (photopheresis) }\end{array}$ & $\begin{array}{l}\text { isolation and sequestration of blood } \\
\text { components } \\
\text { photopheresis therapy }\end{array}$ & $\begin{array}{l}\text { cannulas } \\
\text { blood pump } \\
\text { plasma pump } \\
\text { plasma separator } \\
\text { lipo-sorber } \\
\text { membrane filters } \\
\text { centrifugal components } \\
\text { blood warmer } \\
\text { anticoagulants } \\
\text { regeneration solutions/pumps } \\
\text { valves } \\
\text { pressure sensors }\end{array}$ \\
\hline VAD & $\begin{array}{l}\text { heart failure } \\
\text { bridge to transplant } \\
\text { destination therapy }\end{array}$ & partial or total cardiac support & $\begin{array}{l}\text { cannulas } \\
\text { pump (impellar) } \\
\text { Filters } \\
\text { valves }\end{array}$ \\
\hline $\mathrm{ECCO}_{2} \mathrm{R}$ & $\begin{array}{l}\text { pulmonary hypertension } \\
\text { hypercapnia } \\
\text { high airway pressures } \\
\text { respiratory failure/fatigue } \\
\text { weaning from mechanical ventilation } \\
\text { bridge to transplant }\end{array}$ & $\begin{array}{l}\text { oxygenation and } \mathrm{CO}_{2} \text { removal } \\
\text { independent of the lungs and } \\
\text { pulmonary circulation }\end{array}$ & $\begin{array}{l}\text { cannulas } \\
\text { oxygenator } \\
\mathrm{CO}_{2} \text { exchanger (anticoagulant-coated } \\
\text { diffusion membrane) }\end{array}$ \\
\hline ЕСМO & $\begin{array}{l}\text { Refractory cardiopulmonary } \\
\text { dysfunction } \\
\text { bridge to transplant } \\
\text { arrhytrmia } \\
\text { sepsis } \\
\text { drug overdose } \\
\text { PE } \\
\text { anaphylaxis } \\
\text { failure to wean CPB }\end{array}$ & $\begin{array}{l}\text { partial or total cardiac support and } \\
\text { oxygenation and } \mathrm{CO}_{2} \text { removal }\end{array}$ & $\begin{array}{l}\text { cannulas } \\
\text { oxygenator/membrane } \\
\text { heat exchanger } \\
\text { membrane filtration } \\
\text { pump } \\
\mathrm{CO}_{2} \text { exchanger } \\
\text { reservoir } \\
\text { anticoagulant } \\
\text { pressure monitors }\end{array}$ \\
\hline
\end{tabular}

$\mathrm{CO}_{2}$ : carbon dioxide; CPB: cardiopulmonary bypass; ECMO: extracorporeal membrane oxygenation; HD: hemodialysis; PE: pulmonary embolism; VAD: ventricular assist device. 
Table 2: Extracorporeal circuit related adverse events and associated mechanisms of action

\begin{tabular}{|c|c|c|}
\hline Adverse event & Mechanism of action & References \\
\hline anemia & $\begin{array}{l}\text { contact with foreign material (circuit tubing/cannulas) } \\
\text { increased shear forces } \\
\text { decreased red blood cell deformability } \\
\text { hemolysis }\end{array}$ & [105] \\
\hline thrombocytopenia & $\begin{array}{l}\text { platelet activation } \\
\text { platelet sequestration }\end{array}$ & {$[15,106]$} \\
\hline coagulopathy & $\begin{array}{l}\text { loss/dysfunction of von Willebrand Factor, fibrinogen, complement factors, } \\
\text { coagulation factors } \\
\text { requirement for anticoagulation (e.g. heparin) } \\
\text { disseminated intravascular coagulopathy }\end{array}$ & {$[21,25,61,107]$} \\
\hline inflammation & $\begin{array}{l}\text { increased endothelial permeability } \\
\text { increased susceptibility to infection } \\
\text { reactive oxygen species } \\
\text { myeloperoxidase enzyme activity } \\
\text { complement activation } \\
\text { thrombin } \\
\text { elastase } \\
\text { neutrophil extracellular }\end{array}$ & {$[21,108]$} \\
\hline cardiac dysfunction & $\begin{array}{l}\text { inflammatory cytokine release } \\
\text { arrythmia } \\
\text { myocardial infarction }\end{array}$ & {$[56,108]$} \\
\hline pulmonary injury & cell free hemoglobin & [109] \\
\hline gastrointestinal bleeding & $\begin{array}{l}\text { abnormal cell growth - arterio-venous malformations } \\
\text { continuous as opposed to pulsatile flows }\end{array}$ & {$[3,25,110]$} \\
\hline brain injury & $\begin{array}{l}\text { cannula positioning leading to ischemia } \\
\text { embolic/hemorrhagic stroke }\end{array}$ & {$[3,21,25]$} \\
\hline kidney injury & $\begin{array}{l}\text { low flow states } \\
\text { cardio-renal syndrome }\end{array}$ & {$[21,56]$} \\
\hline
\end{tabular}

adverse events. EVs are a global designation for a range of small $(<1 \mu \mathrm{m})$ cell derived lipid vesicular structures with differing sizes, compositions and origins. Within the EV hierarchy, vesicles can be discriminated in part based on specific triggers for their formation and the composition of their membranes and intravesicular cargos (reviewed in part in $[16,17]$ ). EVs can be further subdivided by size as apoptotic bodies that are larger than microparticles (MPs) /microvesicles (MVs) which are in turn larger than exosomes respectively. First, apoptotic bodies are formed during apoptosis and are physically large EVs typically $>400 \mathrm{~nm}-1000 \mathrm{~nm}$ rivalling platelets in terms of size, but unlike platelets contain histones and genomic DNA. Apoptotic bodies are formed from cellular fragmentation during apoptosis. Second, MP/MV sized EVs typically form from sections of externalized inner cell lipid bilayer membrane involving lipid rafts, especially under conditions of cellular stress or activation and are typically 100-1000 nm). Under usual conditions, membrane asymmetry is maintained by enzymes such as flippases, floppases and scrambles, yet when EVs are formed the activity of these enzymes is altered, in part due to increased intracellular calcium which culminate in cellular contraction, membrane lipid changes and blebbing of EVs [17]. EVs can originate from many different types of parent cells such as: endothelial cells (e-EVs), endothelial progenitor cells (EPC-EVs), leukocytes (1-EVs), platelets (p-EVs), erythrocytes (r-EVs), smooth muscle cells (smc-EVs), monocytes (m-EVs) and granulocytes (neutrophils; g-EVs). MPs can be studded with outer membrane surface markers which in part facilitate characterizing their parent cells of origin. Third, exosomes form from extracellular release of intracellular mutivesicular bodies and are typically smaller, commonly less than $100 \mathrm{~nm}$ in diameter. As opposed to MPs, exosomes express a more concentrated endowment of heat shock proteins, tetraspanins such as CD63 or CD9 and have considerable acetyl-cholinesterase activity [18]. Exosomes are thought to form intracellularly at multivesicular bodies which merge and are released outside the cell membrane [17]. EVs such as MPs are of interest in an ever-increasing number of research fields (such as oncology) due to their potential duality of being biomarkers but also potential effectors of disease [19]. EVs are of interest as they may mediate physiological changes occurring with ExCor/ ECLS use, as will be described later this review. Emerging strategies of modify EV-functions including: reducing $\mathrm{EV}$ 
Table 3: Extracorporeal circuits (ECLS/ExCor systems) lead to changes in systemic flows, shear stresses and may require anticoagulation

\begin{tabular}{|c|c|c|c|}
\hline Circuit & Systemic flows & Shear stresses possible with ExCor/ECLS use & $\begin{array}{c}\text { Systemic } \\
\text { anticoagulant }\end{array}$ \\
\hline $\begin{array}{l}\text { normal circulation } \\
\text { (no ExCor/ECLS) }\end{array}$ & $\begin{array}{c}\text { pulsatile, } \\
2.6-4.2 \mathrm{~L} / \mathrm{min} / \mathrm{m}^{2}\end{array}$ & straight arteries laminar flow $1.5-1.68 \mathrm{~N} / \mathrm{m}^{2}[111,112]$ & no \\
\hline HD & $\begin{array}{c}\text { pulsatile } \\
0.5-0.8 \mathrm{~L} / \mathrm{min}[113]\end{array}$ & $114 \mathrm{~N} / \mathrm{m}^{2}[114]$ & yes \\
\hline CPB & $\begin{array}{l}\text { continuous or pulsatile } \\
1.75 \text { to } 3.5 \mathrm{~L} / \mathrm{min} / \mathrm{m}^{2}\end{array}$ & $994 \mathrm{~N} / \mathrm{m}^{2}[115]$ & yes \\
\hline apheresis & $\begin{array}{c}\text { pulsatile } \\
0.06-0.24 \mathrm{~L} / \mathrm{min}[116]\end{array}$ & not reported & yes \\
\hline VAD & $\begin{array}{l}\text { continuous or pulsatile } \\
3.5-10 \mathrm{~L} / \mathrm{min}[117]\end{array}$ & $80-300 \mathrm{~N} / \mathrm{m}^{2}[22,118]$ & yes \\
\hline $\mathbf{E C C O}^{2} \mathrm{R}$ & $\begin{array}{c}\text { pulsatile } \\
1-15 \mathrm{~L} / \mathrm{min}[119]\end{array}$ & not reported & no \\
\hline ECMO & $\begin{array}{l}\text { continuous } \\
4-8 \mathrm{~L} / \mathrm{min}\end{array}$ & $2 \mathrm{~N} / \mathrm{m}^{2}[120]$ & yes \\
\hline
\end{tabular}

CPB: cardiopulmonary bypass; ECMO: extracorporeal membrane oxygenation; HD: hemodialysis; VAD: ventricular assist device.

numbers or selectively remove detrimental- or increasing beneficial-EVs as well as pharmacological strategies to modify EVs to make them less injurious will be discussed in the review. This review will predominantly discuss the interactions of EVs primarily resembling MP/MVs in relation to ExCor/ECLS as the literature is essentially devoid of descriptions of ExCor/ECLS effects on apoptotic bodies or exosomes.

\section{EVS ARE ASSOCIATED WITH EXCOR/ ECLS USE}

EVs are plausible candidate biomarkers for ExCor/ ECLS adverse events due to their association with ExCor/ ECLS use. As we discuss later in detail, a challenge affecting the interpretation of many EV-ExCor/ECLS studies is the general problem of differentiating between $\mathrm{EV}$ formation due to ExCor/ECLS as opposed to disease progression. A range of ExCor/ECLS systems including CPB, ECMO, VADs, HD and plasmapheresis systems have been shown to be associated with elevated levels of EVs. In addition to ExCor/ECLS systems, their adjuncts, such as pericardial suction may be rich sources of EVs - up to ten-fold higher than pre-operative blood levels $[20,21]$ - which in certain cases get re-transfused to the patient. The number and types of EVs depends at least in part on the different components found in various circuits (Table 1). The scarcity of EV studies related to individual components within ECLS/ExCor systems necessitates that for the purpose of the present review, we have primarily presented EV studies associated with ECLS/ExCor modalities rather than circuit components.

Within individual ExCor/ECLS systems, EV numbers and subtypes may vary depending on the specific design aspects of certain ExCor/ECLS systems leading to increased shear stresses and thus, to EV formation (Figure 1). For example, high shear (up to $300 \mathrm{~N} / \mathrm{m}^{2}$ ) models simulating ExCor/ECLS cause increased formation of p-EVs [22]. When present in ExCor/ECLS systems, oxygenators are a specific site of EV generation as $\mathrm{p}-\mathrm{EV}$ levels were shown to be higher post- as compared to preoxygenator [23]. Beyond the oxygenator, pumps which actively propel blood through ECLS systems can lead to variable EV formation. Using in vitro swine blood, a recent study demonstrated higher p-EVs in centrifugal compared with roller-head pump systems at comparable flows of 0.3 L/minute [24]. Importantly, susceptibility of EV formation seems to depend on the cell types exposed to ExCor/ECLS systems. Within elevated shear stress environments such as seen with ExCor/ECLS use, platelets are considerably more vulnerable to activation and fragmentation at elevated shears $\left(10-25 \mathrm{~N} / \mathrm{m}^{2}\right)$ than erythrocytes or leukocytes [25-27]. The shear-induced generation of p-EVs in ExCor/ECLS is considered to occur analogous to altered circulatory flow conditions in other non- ExCor/ECLS contexts, as e.g. mitral valve disease has been shown to 
Table 4: Biomarkers predicting adverse events during extracorporeal circuits

\begin{tabular}{|c|c|}
\hline Adverse event & Biomarker \\
\hline hemolysis & $\begin{array}{l}\downarrow \text { haptoglobin }[121] \\
\uparrow \text { free-hemoglobin }[3,122] \\
\uparrow \text { fibrin stranding } \\
\downarrow \text { hemoglobin }\end{array}$ \\
\hline inflammation & $\uparrow$ platelet - leukocyte aggregates, NETS $[56,108]$ \\
\hline ischemia reperfusion & $\begin{array}{l}\uparrow \text { lipid peroxidation }[123] \\
\uparrow \text { protein carbonylation }[123]\end{array}$ \\
\hline endothelial dysfunction & $\begin{array}{l}\uparrow \text { circulating endothelial cells }[59,71,124] \\
\uparrow \text { circulating endothelial cell progenitors }[58,71,124] \\
\uparrow \text { circulating soluble E-selectin }[25]\end{array}$ \\
\hline platelet activation & $\begin{array}{l}\uparrow \mathrm{sCD} 40 \mathrm{~L}[25] \\
\uparrow \text { circulating soluble P-selectin }[25,56] \\
\downarrow \text { platelet count } \\
\downarrow \text { platelet function testing }\end{array}$ \\
\hline coagulation & $\begin{array}{l}\uparrow \mathrm{TF}[25] \\
\downarrow \text { large vWF multimers }[3,25] \\
\downarrow \text { AT3, }\end{array}$ \\
\hline infection & $\begin{array}{l}\uparrow \text { procalcitonin }[125] \\
\uparrow \mathrm{CRP}\end{array}$ \\
\hline mortality & $\begin{array}{l}\uparrow \text { ALT/billirubin [126] } \\
\downarrow \text { urine output [127] }\end{array}$ \\
\hline
\end{tabular}

ALT: alanine aminotransferase; AT3: anti-thrombin III; CPB: cardiopulmonary bypass; CRP: C reactive protein; TF: tissue factor; vWF: von Willebrand factor.

have twice the circulating p-EV levels compared to normal controls [28]. Beyond platelets, other cell types such as the endothelium have shown associations with EV formation under the altered flow conditions imposed by ExCor/ECLS. Part of the endothelial response to altered flow within the circulation is to increase endothelial-EV populations. An example of this concept is an experiment with a blood pressure cuff inflated to $220 \mathrm{mmHg}$ for 20 minutes on one arm showed acute increases in e-EVs relative to the other arm with no cuff in healthy volunteers [29]. Though in this instance transient ischemia then reperfusion may also have additionally contributed to increased e-EVs which is different from typical extracorporeal circuit flows. Beyond altered shear rates, colder temperatures are associated with higher EV levels [27, 30] which are pertinent for ExCor/ECLS systems such as CPB during bypass or deep hypothermic circulatory arrest. As such ExCor/ECLS use often leads to higher levels of EVs of different parental cell origins, to differing degrees depending on the individual components of the ExCor/ECLS systems in question.

\section{EVS INCREASE DURING THE USE OF EXCOR/ECLS}

Studies examining EVs in ExCor/ECLS have shown differences in EV numbers and subtypes over time
(Tables 5-8). The half-life of EVs in the circulation is not well defined under physiological conditions let alone in conjunction with ExCor/ECLS use. Published estimates describe short circulating half-lives of five to ten minutes for $\mathrm{p}-\mathrm{EV} \mathrm{s}$ in the circulation of rabbits [31, 32]. Observations of $\mathrm{p}-\mathrm{EVs}$ kinetics in humans receiving exogenous EVs from blood products show EVs may circulate longer in the order of hours [33]. This is of relevance considering many patients with ExCor/ECLS require transfusion, which may be a confounding source of EVs when EVs are used as a biomarker. ExCor/ ECLS may further complicate kinetic measurements of EVs as they may adhere to the foreign material of the ExCor/ECLS systems. Despite possible sequestration of EVs on foreign materials, or increases related to transfusion, there is a distinct increase in EVs associated with initiation of ExCor/ECLS use. In a pig CPB model, increased $\mathrm{p}-\mathrm{EV} \mathrm{s}$ were evident as early as 10 minutes after onset of CPB [23]. Just as there is a rapid onset, there also appears to be a fairly rapid temporal resolution of elevated p-EV levels post CPB in less than 1 hour $[23,34]$. The transient nature of the EV response is in agreement with dynamic changes in EVs seen with other vascular stressors such as percutaneous coronary catheter procedures which trigger transiently increased levels of circulating e-, p- and r-EVs for 
around an hour due to endothelial traumatization by vascular sheaths and wires (with instrumentation and tubing notably resembling what is required for insertion and maintenance of ExCor/ECLS) but return to lower pre-procedure levels after one hour [35]. Notably, however, increases in p-EVs during CPB have not been unanimously confirmed by all studies, as e.g. a study examining 71 patients requiring $\mathrm{CPB}$ showed signs of complement activation but no accumulation of $\mathrm{p}-\mathrm{EV} \mathrm{s}$ post CPB [36]. The lack of increase in this specific study may, however, be attributable to the sole measurement of phosphatidylserine (PS+) EVs which would not have captured non-PS+ EV increases over time from CPB.

Trends of increasing EV numbers over time were seen at 6 and 24 hours in in vitro tests involving prolongedECLS such as VADs (VentrAssist IRBP, RotaFlow CP and CentriMag) under constant hemodynamic conditions and flows $(5 \mathrm{~L} / \mathrm{min})[3,37]$ (Table 6$)$. While there is general consensus that VADs cause an acute increase in circulating EV levels, long-term effects are more variable. At 3 months post-VAD insertion, $r-, 1-$ and e-EVs were found to be increased in patients with HeartWare LVADs compared to VAD free controls [38]. Data on EV numbers at 3 months post VAD insertion are, however, not consistent in the literature in as much as other studies found no difference in PS+ EVs after 3 months in comparison to pre-VAD baseline levels [39] while a study of 30 patients with HEART MATE II units showed decreased PS + EV levels at 3 months in comparison to pre-VAD placement [40]. The authors of these studies showing unchanged or lower EV numbers theorized patients likely have improved hemodynamics post-VAD implantation. Another possible explanation for these discrepancies again lies in methodological differences of the latter two studies which only examined PS + EVs, which represent only a fraction of the total EVs found in the circulation. An additional study examining LVADs showed a reduction in EV numbers at 3 months post-insertion compared with baseline at time of VAD placement which then returned to baseline levels at 6 months post insertion [41]. Though the changes were not statistically significant, in this study the authors theorized that initially at 3 months EVs may have fallen due to improved perfusion, yet after 6 months the potentially high shear rates may have accelerated EV
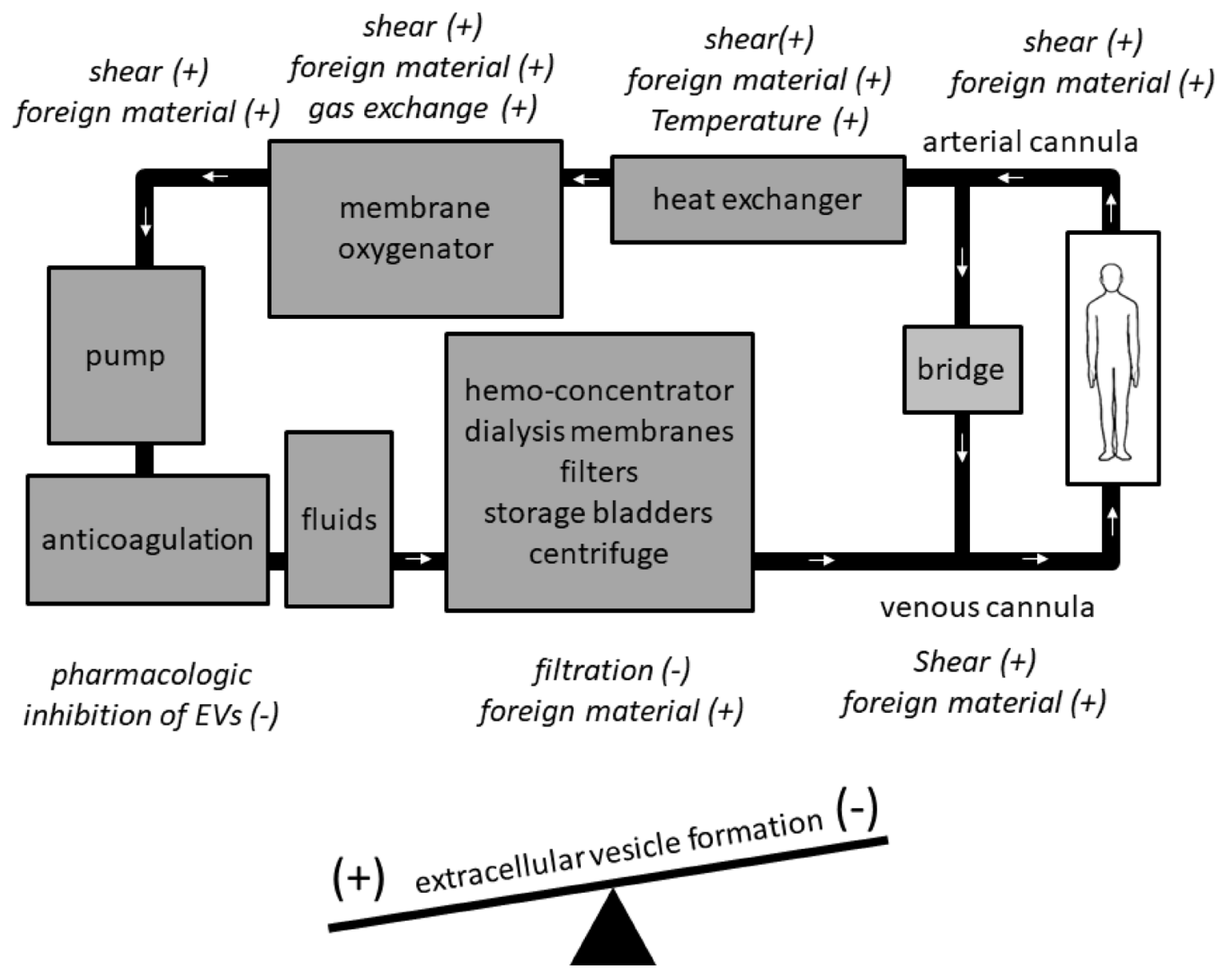

Figure 1: Specific components of extracorporeal circuits have the potential to increase $(+)$ or decrease $(-)$ extracellular vesicle (EV) levels. 
Table 5: Cardiopulmonary support with extracorporeal circuits (ECLS/ExCor systems) includes CPB and ECMO

\begin{tabular}{|c|c|c|c|c|c|c|}
\hline $\begin{array}{l}\text { Circuit type } \\
\text { Observations }\end{array}$ & $N$ & $\begin{array}{l}\text { CARDIAC } \\
\text { EV phenotype }\end{array}$ & $\begin{array}{l}\mathrm{EV} \text { isolation } \\
\text { technique }\end{array}$ & $\begin{array}{l}\text { Detection } \\
\text { technique }\end{array}$ & Population & Reference \\
\hline $\begin{array}{l}\text { CPB } \\
\text { pericardial blood } \\
\text { contains elevated levels } \\
\text { of EVS }\end{array}$ & 13 & $\begin{array}{c}\text { C1q C4 C3 } \\
\text { complement bound } \\
\text { EVs }\end{array}$ & $\begin{array}{l}\text { citrated plasma } \\
1550 \mathrm{~g} \times 20 \\
\text { minutes } \\
-80 \mathrm{C} \text { thawed } \\
19000 \mathrm{~g} \times 30 \\
\text { minutes }\end{array}$ & FCM & $\begin{array}{l}\text { adult } \\
\text { cardiac } \\
\text { surgery }\end{array}$ & [21] \\
\hline $\begin{array}{l}\text { CPB } \\
\text { iNO and PGI2 reduced } \\
\text { p-EV levels }\end{array}$ & 41 & CD42 p-EVs & citrated plasma & FCM & $\begin{array}{l}\text { adult } \\
\text { cardiac } \\
\text { surgery }\end{array}$ & [61] \\
\hline $\begin{array}{l}\mathrm{CPB} \\
\text { transient } C P B \\
\text { increased } p-E V S\end{array}$ & 12 & CD61 CD62 p-EVs & citrated plasma & FCM & pigs & [23] \\
\hline $\begin{array}{l}\mathrm{CPB} \\
\text { transient } C P B \\
\text { increased } p-E V S\end{array}$ & 18 & CD61 CD62 p-EVs & citrated plasma & FCM & pigs & [128] \\
\hline $\begin{array}{l}\text { CPB } \\
\text { lower levels of EVs in } \\
\text { CPB compared with } \\
\text { suctioned pericardial } \\
\text { blood }\end{array}$ & $\begin{array}{l}2 \text { females } \\
8 \text { males }\end{array}$ & Annexin V EVs & $\begin{array}{l}\text { citrated plasma } \\
3000 \mathrm{~g} \times 10 \\
\text { minutes } \\
13000 \mathrm{~g} \times 2 \\
\text { minutes }\end{array}$ & ELISA & $\begin{array}{l}\text { adult } \\
\text { cardiac } \\
\text { surgery }\end{array}$ & [20] \\
\hline $\begin{array}{l}\text { Neonatal (ECMO) } \\
\text { Jostra-rotaflow-HL20 } \\
\text { Centrifugal ECMO } \\
\text { created more p-EVS } \\
\text { than roller pump } \\
\text { ECMO }\end{array}$ & 12 & CD61 p-EVs & $\begin{array}{l}\text { heparinized blood } \\
300 \mathrm{~g} \times 10 \\
\text { minutes post } \\
\text { staining }\end{array}$ & FCM & $\begin{array}{l}\text { in vitro } \\
\text { pig }\end{array}$ & [24] \\
\hline $\begin{array}{l}\text { CPB } \\
\text { increased EV levels } \\
\text { in pericardial blood } \\
\text { compared with systemic } \\
\text { blood }\end{array}$ & 6 & $\begin{array}{l}\text { Annexin V EVs } \\
\text { CD61 CD42 p-EVs } \\
\text { CD235 r-EVs } \\
\text { CD14 m-EVs }\end{array}$ & $\begin{array}{l}\text { citrated plasma } \\
1550 \mathrm{~g} \times 15 \\
\text { minutes }\end{array}$ & $\begin{array}{l}\text { FCM } \\
\text { In vitro } \\
\text { Thrombin } \\
\text { assays }\end{array}$ & $\begin{array}{l}\text { adult } \\
\text { cardiac } \\
\text { surgery }\end{array}$ & [72] \\
\hline $\begin{array}{l}\text { CPB } \\
\text { cell saver removed } E V S\end{array}$ & 13 & $\begin{array}{l}\text { Annexin V EVs } \\
\text { CD235 r-EVs } \\
\text { CD61 p-EVs }\end{array}$ & $\begin{array}{l}\text { citrated plasma } \\
1550 \mathrm{~g} \times 20 \\
\text { minutes } \\
17570 \mathrm{~g} \times 30 \\
\text { minutes }\end{array}$ & FCM & $\begin{array}{l}\text { adult } \\
\text { cardiac } \\
\text { surgery }\end{array}$ & [75] \\
\hline $\begin{array}{l}\text { CPB } \\
\text { pericardial blood has } \\
\text { elevated EVs which } \\
\text { upon retransfusion do } \\
\text { not persistently stay } \\
\text { elevated in circulation }\end{array}$ & $\begin{array}{l}2 \text { females } \\
11 \text { males }\end{array}$ & $\begin{array}{l}\text { Annexin V EVs } \\
\text { CD235 r-EVs } \\
\text { CD61 p-EVs }\end{array}$ & $\begin{array}{l}\text { citrated plasma } \\
1550 \mathrm{~g} \times 20 \\
\text { minutes } \\
17570 \mathrm{~g} \times 30 \\
\text { minutes }\end{array}$ & FCM & $\begin{array}{l}\text { adult } \\
\text { cardiac } \\
\text { surgery }\end{array}$ & [129] \\
\hline $\begin{array}{l}\text { CPB } \\
\text { complement complexes } \\
\text { were elevated post CPB } \\
\text { while p-EVs were not }\end{array}$ & $\begin{array}{l}3 \text { females } \\
68 \text { males }\end{array}$ & $\begin{array}{l}\text { Annexin V EVs } \\
\text { CD61 p-EVs }\end{array}$ & $\begin{array}{l}\text { citrated plasma } \\
1550 \mathrm{~g} \times 20 \\
\text { minutes } \\
17570 \mathrm{~g} \times 30 \\
\text { minutes }\end{array}$ & FCM & $\begin{array}{l}\text { adult } \\
\text { cardiac } \\
\text { surgery }\end{array}$ & {$[36]$} \\
\hline
\end{tabular}


ECMO

in vitro experiments

involving prevention of

platelet activation show

decreased p-EV levels

in ECMO circuits after

2 hours

CPB: cardiopulmonary bypass; ECMO: extracorporeal membrane oxygenation; EV: extracellular vesicles; FCM: flow cytometry; m-EV: monocyte-EV; p-EV: platelet-EV; r-EV: red blood cell associated EV.

Table 6: Cardiopulmonary support with extracorporeal circuits (ECLS/ExCor systems) can involve VADs

\begin{tabular}{|c|c|c|c|c|c|c|}
\hline $\begin{array}{l}\text { Circuit type } \\
\text { Observations }\end{array}$ & $N$ & $\begin{array}{c}\text { VAD } \\
\text { EV phenotype }\end{array}$ & $\begin{array}{c}\text { EV } \\
\text { isolation } \\
\text { technique }\end{array}$ & $\begin{array}{l}\text { Detection } \\
\text { technique }\end{array}$ & Population & Reference \\
\hline $\begin{array}{l}\text { LVAD } \\
\text { HMII, HVAD } \\
\text { no change in p-EVs }\end{array}$ & $\begin{array}{l}3 \text { females } \\
17 \text { males }\end{array}$ & $\begin{array}{c}\text { CD62p CD41 } \\
\text { CD42 } \\
\text { p-EVs }\end{array}$ & $\begin{array}{l}\text { PFA fixed } \\
\text { PPP }\end{array}$ & Not disclosed & $\begin{array}{c}\text { adult LVAD } \\
\text { patients }\end{array}$ & [44] \\
\hline $\begin{array}{l}\text { LVAD } \\
\text { Ventrassist IRBP led to more } \\
\text { l-EVs than Rotaflow CP }\end{array}$ & $\begin{array}{c}15 \\
\text { samples }\end{array}$ & CD45 1-EVs & $\begin{array}{l}\text { CPDA cow } \\
\text { blood }\end{array}$ & $\begin{array}{c}\text { EM } \\
\text { FCM } \\
\text { Imaging FCM }\end{array}$ & in vitro & [37] \\
\hline $\begin{array}{l}\text { LVAD } \\
\text { Centrimag CP } \\
\text { increases l-EVs }\end{array}$ & $\begin{array}{c}15 \\
\text { samples }\end{array}$ & CD45 1-EVs & $\begin{array}{l}\text { CDPA cow } \\
\text { blood }\end{array}$ & FCM & in vitro & [3] \\
\hline $\begin{array}{l}\text { LVAD } \\
\text { HMII, Thoratec VAD } \\
\text { Ventrassist, Circulite, } \\
\text { ECMO } \\
\text { increases p- and l-EVs }\end{array}$ & $\begin{array}{l}3 \text { females } \\
9 \text { males }\end{array}$ & $\begin{array}{l}\text { CD31 CD61 p-EVs } \\
\text { CD11b l-EVs } \\
\text { CD62e e-EVs }\end{array}$ & $\begin{array}{c}\text { citrated } \\
\text { plasma } \\
1550 \mathrm{~g} \times \\
15 \mathrm{~min}\end{array}$ & $\mathrm{FCM}$ & $\begin{array}{l}\text { adult LVAD } \\
\text { patients }\end{array}$ & [42] \\
\hline $\begin{array}{l}\text { LVAD } \\
\text { HMII } \\
\text { lower EVs after } 3 \text { months } \\
\text { post-VAD }\end{array}$ & $\begin{array}{l}5 \text { females } \\
25 \text { males }\end{array}$ & Annexin V EVs & $\begin{array}{c}\text { not } \\
\text { disclosed }\end{array}$ & $\begin{array}{l}\text { ELISA } \\
\text { Zymutest EV- } \\
\text { activity test }\end{array}$ & $\begin{array}{l}\text { adult LVAD } \\
\text { patients }\end{array}$ & [40] \\
\hline $\begin{array}{l}\text { LVAD } \\
\text { HMII } \\
\text { EVs higher in patients with } \\
\text { adverse events }\end{array}$ & $\begin{array}{c}17 \\
\text { patients }\end{array}$ & Annexin V EVs & $\begin{array}{c}\text { not } \\
\text { disclosed }\end{array}$ & FCM & $\begin{array}{l}\text { adult LVAD } \\
\text { patients }\end{array}$ & [56] \\
\hline $\begin{array}{l}\text { LVAD } \\
\text { HMII } \\
\text { no differences in EV levels } \\
\text { before and } 3 \text { months after } \\
\text { LVAD insertion }\end{array}$ & 8 males & Annexin V EVs & $\begin{array}{c}\text { not } \\
\text { disclosed }\end{array}$ & $\begin{array}{c}\text { ELISA } \\
\text { Zymutest EV- } \\
\text { activity test }\end{array}$ & $\begin{array}{l}\text { adult LVAD } \\
\text { patients }\end{array}$ & [39] \\
\hline $\begin{array}{l}\text { LVAD } \\
\text { HeartWare continuous flow } \\
\text { elevated p-EVs after } 1 \text { year } \\
4 \text { patients had BiVAD }\end{array}$ & $\begin{array}{l}3 \text { females } \\
25 \text { males }\end{array}$ & $\begin{array}{c}\text { lactadherin CD41 } \\
\text { p-EVs }\end{array}$ & $\begin{array}{l}\text { citrated } \\
\text { PPP }\end{array}$ & FCM & $\begin{array}{l}\text { adult LVAD } \\
\text { patients }\end{array}$ & [43] \\
\hline $\begin{array}{l}\text { LVAD } \\
\text { HMII continuous flow } \\
\text { though not statistically } \\
\text { significant lower EVS } 3 \\
\text { months post-VAD which } \\
\text { returned to baseline at } 6 \\
\text { months post-VAD }\end{array}$ & $\begin{array}{l}5 \text { females } \\
18 \text { males }\end{array}$ & Annexin V EVs & $\begin{array}{c}\text { not } \\
\text { disclosed }\end{array}$ & ELISA & $\begin{array}{l}\text { adult LVAD } \\
\text { patients }\end{array}$ & {$[41]$} \\
\hline
\end{tabular}




\begin{tabular}{|c|c|c|c|c|}
\hline $\begin{array}{l}\text { LVAD } \\
\text { HeartWare } \\
\text { increased EVs with LVAD }\end{array}$ & $\begin{array}{c}10 \\
\text { patients }\end{array}$ & $\begin{array}{c}\text { CD41 Cd31 p-EVs } \\
\text { CD45 l-EVs } \\
\text { CD235 r-EVs } \\
\text { CD62 CD144 } \\
\text { e-EVs } \\
\text { CD31 e-EVS }\end{array}$ & $\begin{array}{c}\text { citrated } \\
\text { plasma } \\
300 \mathrm{~g} \times 15 \\
\mathrm{~min}, 10000 \\
\mathrm{~g} \times 5 \mathrm{~min} \\
\text { twice, } \\
30000 \mathrm{~g} \times \\
90 \mathrm{~min} \\
4^{\circ} \mathrm{C}\end{array}$ & $\begin{array}{c}\text { FCM } \\
\text { ELISA }\end{array}$ \\
\hline
\end{tabular}

CPDA: citrate phosphate dextrose adenine; e-EV: endothelial-EV; EM: electron microscopy; EV: extracellular vesicles; FCM: flow cytometry; HM: HeartMate; l-EV: leukocyte-EV; PFA: paraformaldehyde; p-EV: platelet-EV; PPP : platelet poor plasma; r-EV: red blood cell derived EV.

Table 7: Renal support with extracorporeal circuits (ExCors) includes HD

\begin{tabular}{|c|c|c|c|c|c|c|}
\hline $\begin{array}{l}\text { Circuit type } \\
\text { Observations }\end{array}$ & $N$ & $\begin{array}{c}\text { Dialysis } \\
\text { EV phenotype }\end{array}$ & $\mathrm{EV}$ isolation & $\begin{array}{l}\text { Detection } \\
\text { technique }\end{array}$ & $\begin{array}{c}\text { Patient } \\
\text { population }\end{array}$ & Reference \\
\hline $\begin{array}{l}\text { CVVH } \\
\text { in vitro use of } C V V H \\
\text { can remove e-EVs }\end{array}$ & $\mathrm{n} / \mathrm{a}$ & $\begin{array}{l}\text { Annexin V EVs } \\
\text { CD31 e-EVs }\end{array}$ & $\begin{array}{c}\text { cell culture } \\
145 \mathrm{~g} \times 8 \text { minutes } \\
100000 \mathrm{~g} \times 6 \\
\text { minutes }\end{array}$ & $\mathrm{FCM}$ & $\begin{array}{l}\text { in vitro } \\
\text { Endothelial } \\
\text { cells }\end{array}$ & [74] \\
\hline $\begin{array}{l}\text { HD } \\
\text { dialysis dependent } \\
\text { patients have higher } \\
\text { levels of e-, r-and } \\
\text { p-EVs. e-EV levels } \\
\text { correlated with } \\
\text { vascular function tests }\end{array}$ & $\begin{array}{c}19 \\
\text { females } \\
25 \text { males }\end{array}$ & $\begin{array}{l}\text { Annexin V EVs } \\
\text { CD31 CD144 e-EVs } \\
\text { CD235 r-EVs } \\
\text { CD41 p-EVs } \\
\text { CD3 CD45 l-EVs } \\
\text { Cd11b m-EVs } \\
\text { CD66b g-EVs }\end{array}$ & $\begin{array}{c}\text { citrated plasma } \\
11000 \mathrm{~g} \times 2 \text { minutes } \\
13000 \mathrm{~g} \times 45 \\
\text { minutes } \\
20500 \mathrm{~g} \mathrm{x} 20 \\
\text { minutes }\end{array}$ & FCM & $\begin{array}{l}\text { adult } \\
\text { ESRD } \\
\text { patients }\end{array}$ & [67] \\
\hline $\begin{array}{l}\text { HD } \\
\text { EMP levels correlated } \\
\text { with cardiovascular } \\
\text { risk in ESRD patients }\end{array}$ & $\begin{array}{c}30 \\
\text { females } \\
51 \text { males }\end{array}$ & $\begin{array}{c}\text { Annexin V } \\
\text { CD31 CD144 e-EVs } \\
\text { CD11b l-EVs } \\
\text { CD41 CD31 p-EVs } \\
\text { CD235 RMP }\end{array}$ & $\begin{array}{c}\text { citrated plasma } \\
500 \mathrm{~g} \times 15 \text { minutes } \\
14000 \mathrm{~g} \times 5 \text { minutes }\end{array}$ & FCM & $\begin{array}{l}\text { adult } \\
\text { ESRD } \\
\text { patients }\end{array}$ & {$[55]$} \\
\hline $\begin{array}{l}\text { HD } \\
\text { shear stress varies } \\
\text { inversely to EMP levels }\end{array}$ & $\begin{array}{c}14 \\
\text { females } \\
20 \text { males }\end{array}$ & $\begin{array}{l}\text { CD41 CD31 p-EVs } \\
\text { CD31 CD144 e-EVs }\end{array}$ & $\begin{array}{c}\text { citrated plasma } \\
11000 \mathrm{~g} \times 2 \text { minutes } \\
13000 \mathrm{~g} \times 45 \\
\text { minutes } \\
20500 \mathrm{~g} \times 20 \\
\text { minutes }\end{array}$ & FCM & $\begin{array}{l}\text { adult } \\
\text { ESRD } \\
\text { patients }\end{array}$ & [78] \\
\hline $\begin{array}{l}\text { HD } \\
\text { g- and p-EVs } \\
\text { increased with HD }\end{array}$ & $\begin{array}{c}22 \\
\text { females } \\
18 \text { males }\end{array}$ & $\begin{array}{l}\text { CD41 p-EVs } \\
\text { CD66b g-EVs }\end{array}$ & $\begin{array}{c}\text { citrated plasma } \\
150 \mathrm{~g} \times 20 \text { minutes } \\
300 \mathrm{~g} \times 20 \text { minutes } \\
15000 \mathrm{~g} \times 30 \\
\text { minutes with EDTA } \\
\text { treatment }\end{array}$ & FCM & $\begin{array}{l}\text { adult } \\
\text { ESRD } \\
\text { patients }\end{array}$ & [48] \\
\hline $\begin{array}{l}\text { HD } \\
\text { e-EV levels were } \\
\text { higher in HD patients } \\
\text { compared with pre-HD } \\
\text { or PD patients }\end{array}$ & $\begin{array}{c}6 \\
\text { females } \\
6 \text { males }\end{array}$ & CD144 CD146 e-EVs & $\begin{array}{c}\text { citrated plasma } \\
1500 \mathrm{~g} \times 15 \text { minutes } \\
13000 \mathrm{~g} \times 2 \text { minutes }\end{array}$ & FCM & $\begin{array}{l}\text { pediatric } \\
\text { ESRD } \\
\text { patients }\end{array}$ & {$[52]$} \\
\hline
\end{tabular}




\begin{tabular}{|c|c|c|c|c|c|}
\hline $\begin{array}{l}\text { HD } \\
\text { patients having HD } \\
\text { had elevated levels } \\
\text { of all EVs examined. } \\
\text { Patients had elevations } \\
\text { in p-EVs after HD } \\
\text { sessions }\end{array}$ & $\begin{array}{c}14 \\
\text { females } \\
16 \text { males }\end{array}$ & $\begin{array}{c}\text { Annexin V EVs } \\
\text { CD41 p-EVs } \\
\text { CD144 CD146 e-EVs } \\
\text { CD45 l-EVs }\end{array}$ & $\begin{array}{c}\text { citrated plasma } \\
1500 \mathrm{~g} \times 15 \text { minutes } \\
13000 \mathrm{~g} \times 2 \text { minutes }\end{array}$ & $\mathrm{FCM}$ & $\begin{array}{l}\text { adult } \\
\text { ESRD } \\
\text { patients }\end{array}$ \\
\hline $\begin{array}{l}\text { HD } \\
\text { patients having HD } \\
\text { show indirect evidence } \\
\text { of increased p-EVs } \\
\text { levels during dialysis }\end{array}$ & $\begin{array}{c}4 \\
\text { females } \\
3 \text { males }\end{array}$ & CD41 CD62 p-EVs & $\begin{array}{c}\text { citrated plasma } \\
180 \mathrm{~g} \times 20 \text { minutes } \\
13000 \mathrm{~g} \times 2 \text { minutes }\end{array}$ & $\mathrm{FCM}$ & $\begin{array}{l}\text { adult } \\
\text { ESRD } \\
\text { patients }\end{array}$ \\
\hline $\begin{array}{l}\text { HD } \\
\text { patients having HD } \\
\text { had elevated levels } \\
\text { of } \mathrm{e}-\mathrm{EV} \text { shich } \\
\text { correlated with } \\
\text { endothelial progenitor } \\
\text { cell levels }\end{array}$ & $\begin{array}{c}18 \\
\text { females } \\
20 \text { males }\end{array}$ & CD31 e-EVs & $\begin{array}{c}\text { citrated plasma } \\
1500 \mathrm{~g} \times 15 \text { minutes } \\
13000 \mathrm{~g} \times 2 \text { minutes }\end{array}$ & FCM & $\begin{array}{l}\text { adult } \\
\text { ESRD } \\
\text { patients }\end{array}$ \\
\hline $\begin{array}{l}\text { HD } \\
\text { patients having HD } \\
\text { have elevated } \mathrm{e}-\mathrm{EV} \\
\text { levels in comparison } \\
\text { to non-HD ESRD and } \\
\text { control patients }\end{array}$ & $\begin{array}{l}11 \\
\text { females } \\
4 \text { males }\end{array}$ & $\begin{array}{l}\text { Annexin V EVs } \\
\text { CD31 e-EVs }\end{array}$ & $\begin{array}{c}\text { citrated plasma } \\
13000 \mathrm{~g} \times 5 \text { minutes }\end{array}$ & FCM & $\begin{array}{l}\text { adult } \\
\text { ESRD } \\
\text { patients }\end{array}$ \\
\hline $\begin{array}{l}\text { HD } \\
\text { on-line hemofiltration } \\
\text { for HD patients may } \\
\text { reduce e-EV levels }\end{array}$ & $\begin{array}{c}4 \\
\text { females } \\
11 \text { males }\end{array}$ & $\begin{array}{l}\text { Annexin V EVs } \\
\text { CD31 e-EVs }\end{array}$ & $\begin{array}{c}\text { citrated plasma } \\
13000 \mathrm{~g} \times 5 \text { minutes }\end{array}$ & FCM & $\begin{array}{l}\text { adult } \\
\text { ESRD } \\
\text { patients }\end{array}$ \\
\hline $\begin{array}{l}\text { HD } \\
\text { patients having HD } \\
\text { have elevated levels of } \\
\text { EVs }\end{array}$ & $\begin{array}{c}5 \\
\text { females } \\
5 \text { males }\end{array}$ & $\begin{array}{c}\text { Annexin V EVs } \\
\text { CD41 CD62p CD63 } \\
\text { CD61 p-EVs } \\
\text { CD235 r-EVs } \\
\text { CD144 CD62e CD54 } \\
\text { CD106 e-EVs } \\
\text { CD45 CD66e CD20 } \\
\text { CD8 CD4 CD15 } \\
\text { l-EVs } \\
\text { CD66b g-EVs } \\
\text { CD14 m-EVs } \\
\text { TF EVs }\end{array}$ & $\begin{array}{c}\text { citrated plasma } \\
1550 \mathrm{~g} \times 20 \text { minutes }\end{array}$ & $\begin{array}{l}\text { FCM } \\
\text { EV } \\
\text { thrombin } \\
\text { generation } \\
\text { assay }\end{array}$ & $\begin{array}{l}\text { adult } \\
\text { ESRD } \\
\text { patients }\end{array}$ \\
\hline $\begin{array}{l}\text { HD } \\
\text { patients having HD or } \\
\text { PD have elevated levels } \\
\text { of pro-coagulant EVs }\end{array}$ & $\begin{array}{c}10 \\
\text { females } \\
10 \text { males }\end{array}$ & $\begin{array}{l}\text { CD144 e-EVs } \\
\text { CD42b p-EVs }\end{array}$ & $\begin{array}{c}\text { citrated } \\
\text { Plasma } \\
1500 \mathrm{~g} \times 15 \text { minutes } \\
\text { twice then } 13000 \mathrm{~g} \times \\
2 \text { minutes then } \\
18000 \mathrm{~g} \times 30 \\
\text { minutes }\end{array}$ & $\begin{array}{l}\text { NTA } \\
\text { WB } \\
\text { EM } \\
\text { EV } \\
\text { thrombin } \\
\text { generation } \\
\text { assay }\end{array}$ & $\begin{array}{l}\text { adult } \\
\text { ESRD } \\
\text { patients }\end{array}$ \\
\hline
\end{tabular}




\begin{tabular}{|c|c|c|c|c|c|}
\hline $\begin{array}{l}\text { HD } \\
\text { reductions in e-EVs } \\
\text { were greater with } \\
\text { HFR than online } \\
\text { hemofiltration }\end{array}$ & $\begin{array}{c}7 \\
\text { females } \\
10 \text { males }\end{array}$ & $\begin{array}{c}\text { CD31 e-EVs } \\
\text { Annexin V EVs }\end{array}$ & not disclosed & FCM & $\begin{array}{c}\text { adult } \\
\text { ESRD } \\
\text { patients }\end{array}$ \\
\hline $\begin{array}{l}\text { HD } \\
\text { increased p- and } \\
\text { e-EVs vascular } \\
\text { access failure which } \\
\text { correlates with ExCor } \\
\text { stenosis }\end{array}$ & $\begin{array}{c}52 \\
\text { females } \\
30 \text { males }\end{array}$ & $\begin{array}{l}\text { CD31/CD51 e-EVs } \\
\text { CD31 CD41 p-EVs }\end{array}$ & $\begin{array}{l}\text { citrated plasma } 160 \\
\mathrm{~g} \times 5 \mathrm{~min} 4^{\circ} \mathrm{C} \text { then } \\
1200 \mathrm{~g} \times 6 \mathrm{~min} 4^{\circ} \mathrm{C}\end{array}$ & FCM & $\begin{array}{l}\text { adult } \\
\text { ESRD } \\
\text { patients }\end{array}$ \\
\hline
\end{tabular}

CVVH: continuous veno-veno hemofiltration; EM: electron microscopy; e-EV: endothelial-EV; EV: extracellular vesicle; FCM: flow cytometry; g-EV: granulocyte-EV; HD: hemodialysis; HFR: hemofiltration with endogenous reinfusion; 1-EV: leukocyte-EV; m-EV: monocyte-EV; NTA: nanoparticle tracking analysis; PD: peritoneal dialysis; p-EV: platelet-EV; r-EV: red blood cell derived EV; WB Western Blot.

Table 8: Apheresis circuits are extracorporeal circuits (ExCors) indicated for a wide range of diseases including intoxications and autoimmune conditions

\begin{tabular}{|c|c|c|c|c|c|c|}
\hline $\begin{array}{l}\text { Circuit type } \\
\text { Observations }\end{array}$ & $N$ & EV phenotype & $\begin{array}{c}\text { Apheresis } \\
\text { EV isolation }\end{array}$ & $\begin{array}{l}\text { Detection } \\
\text { techniques }\end{array}$ & $\begin{array}{c}\text { Patient } \\
\text { population }\end{array}$ & Reference \\
\hline $\begin{array}{l}\text { apheresis circuit } \\
\text { lowered } E V S\end{array}$ & $\begin{array}{l}9 \text { males } \\
3 \\
\text { females }\end{array}$ & $\begin{array}{l}\text { Annexin V EVs } \\
\text { CD41 p-EVs } \\
\text { CD144 e-EVs } \\
\text { CD11b m-EVs } \\
\text { CD235 r-EVs }\end{array}$ & $\begin{array}{c}\text { citrated plasma } \\
1509 \mathrm{~g} \times 10 \mathrm{~min} \\
100000 \mathrm{~g} \times 60 \mathrm{~min}\end{array}$ & $\begin{array}{c}\text { NTA } \\
\text { TRPS } \\
\text { FCM } \\
\text { EV fatty } \\
\text { acids } \\
\text { EV IIa } \\
\text { generation }\end{array}$ & $\begin{array}{l}\text { adult FH } \\
\text { patients }\end{array}$ & {$[76]$} \\
\hline $\begin{array}{l}\text { leukopheresis } \\
\text { increased p-EVs }\end{array}$ & $\begin{array}{l}8 \text { males } \\
4 \\
\text { females }\end{array}$ & CD42a p-EVs & $\begin{array}{l}\text { citrated plasma } \\
200 \mathrm{~g} \times 10 \mathrm{~min} \\
1000 \mathrm{~g} \times 15 \mathrm{~min}\end{array}$ & FCM & $\begin{array}{l}\text { adult } \\
\text { malignant } \\
\text { lymphoma } \\
\text { patients }\end{array}$ & [130] \\
\hline $\begin{array}{l}\text { leukopheresis } \\
\text { decreased p-EVs and } \\
\text { increased g-EVs }\end{array}$ & $\begin{array}{c}6 \\
\text { females }\end{array}$ & $\begin{array}{c}\text { Annexin V EVs } \\
\text { CD61 CD42a } \\
\text { p-EVs } \\
\text { CD66 CD16 } \\
\text { g-EVs }\end{array}$ & $\begin{array}{c}\text { EDTA plasma } \\
1600 \mathrm{~g} \times 20 \mathrm{~min} \\
-70 \mathrm{C} \text { freeze, thawed } \\
17000 \mathrm{~g} \times 20 \mathrm{~min}\end{array}$ & $\begin{array}{l}\text { EM } \\
\text { FCM }\end{array}$ & $\begin{array}{l}\text { adult RA } \\
\text { patients }\end{array}$ & [80] \\
\hline $\begin{array}{l}\text { plasmapheresis } \\
\text { increased p-EVs }\end{array}$ & $\begin{array}{l}6 \text { males } \\
6 \\
\text { females }\end{array}$ & $\begin{array}{l}\text { PAC-1 CD62p } \\
\text { CD61 p-EVs }\end{array}$ & citrated plasma & FCM & $\begin{array}{c}\text { adult } \\
\text { volunteers }\end{array}$ & [131] \\
\hline $\begin{array}{l}\text { plateletpheresis } \\
\text { produce differing } \\
\text { levels of } \mathrm{p}-\mathrm{EVs} \\
\text { in their platelet } \\
\text { concentrates }\end{array}$ & $\begin{array}{c}42 \\
\text { platelet } \\
\text { samples }\end{array}$ & CD61 p-EVs & citrated plasma & $\begin{array}{l}\text { FCM } \\
\text { NTA }\end{array}$ & adult donors & [83] \\
\hline
\end{tabular}

EM: electron microscopy; Extracellular vesicle (EV); FCM: flow cytometry; FH: familial hypercholesterolemia; g-EV: granulocyte-EV; m-EV: monocyte-EV; NTA: nanoparticle tracking analysis, p-EV: platelet-EV; RA: rheumatoid arthritis; r-EV: red blood cell derived EV; TRPS: tunable resistive pulse sensing. 
production back to levels similar to baseline. Interpretation of this study in comparison to others is challenging due to the absence of a control group (non-VAD patients) and lack of phenotypic investigation of the EV populations beyond enumeration of PS expressing EVs. Yet a study of 12 patients on extracorporeal assist devices for approximately 5 months, of which 11 had VADs showed elevated counts of p-, 1- and e-EVs compared with control patient who did not have these devices which corroborates a longer-term increase in EVs ( $>3$ months) [42]. Finally, extrapolating the general trend of increasing number of EVs over time seen in studies ranging from seconds, hours to months, after a year or longer two studies have found elevations in $\mathrm{p}$-EVs relative to baseline initiation of VAD support in LVAD and BiVAD (/HeartMate II or Heartware) assisted patients [43, 44]. Taken together, EV numbers are commonly elevated in the circulation of patients who are supported by ExCor/ECLS, with transitory elevations in the case of temporary ExCor/ECLS, and subtler yet progressive increases in case of prolonged-ExCor/ECLS.

\section{LEVELS OF EVS ARE PROPORTIONAL TO DISEASE SEVERITY}

Sick patients, such as those requiring hemodialysis, often have baseline inflammation (prior to ExCor/ ECLS exposure) from chronic renal dysfunction which, independent of ExCor/ECLS use, are associated with elevated levels of circulating EVs. Even more so than in patients with CPBs, ECMO, or VADs, it is therefore critical to distinguish whether EV levels in ExCor/ECLS systems, such as with HD patients (Table 7), are elevated as a result of ExCor/ECLS adverse events or simply as a result of the underlying disease and a reflection of its severity. Of interest, healthy controls have lower levels of e-, p-, g- or PS+-EVs compared to end stage renal disease (ESRD) patients [45-53], which are in turn lower than most accounts of ESRD patients requiring $\operatorname{HD}[48,51,52]$.

As such, a current limitation of interpreting EV studies is the inability to definitively determine if EVs are related to pre-existing inflammatory conditions or the use of ExCor/ECLS systems especially when control groups (healthy individuals or patients with pre-existing inflammation without ExCor/ECLS) are not available for comparison to the ECLS/ExCor patients.

\section{EVS ARE ASSOCIATED WITH ADVERSE EVENTS PERTINENT TO EXCOR/ECLS USE}

For biomarkers of ExCor/ECLS related adverse events, there are presently limitations to obtaining prompt meaningful estimates of ExCor/ECLS related inflammation and apoptosis or an accurate means of predicting pending complications during ExCor/ECLS use. An emerging strategy of monitoring these parameters is the study of EVs in the circulations of ExCor/ECLS coupled patients. EVs have been shown to correlate with disease severity and outcomes such as cardiovascular risk; for example, e-EVs correlate with vascular dysfunction in patients with chronic inflammation such as diabetics when compared to healthy controls [54]. E-EVs have also been shown to correlate well with mortality within a high cardiovascular risk group of end stage renal disease (ESRD) patients [55]. Beyond their association with the disease processes which lead patients to requiring ExCor/ECLS, there is growing evidence of the capacity of EVs to act as biomarkers specifically for ExCor/ ECLS adverse events. E-, 1-, p- and r-EVs were examined from 81 patients with ESRD dialyzed three times per week 4-6 hours per session with high permeability membranes (AN69 and polysulphone); and specifically, elevated e-EV levels emerged as a key predictor of cardiovascular mortality within this population [55]. Moreover, PS + EV counts are higher in VAD-patients with cardiac and gastrointestinal adverse events compared with complication-free VAD patients [56]. A study of 17 patients 3 months post-VAD insertion and 10 nonVAD control patients showed that VAD patients with complications had higher PS + EV counts compared with complication-free VAD patients which were again higher than non-VAD controls [56, 57]. Similarly, elevations of e-EVs have correlative association with adverse events such as cardio-renal complications in a variety of ExCor/ ECLS [39, 47, 55, 56, 58, 59]. E-EVs have been proposed to serve as a biomarker for arterial stiffness in pediatric HD patients, as pulse wave velocity, an accepted measure of arterial stiffness, correlated with CD144+ e-EV levels that were higher in HD patients compared with non-HD controls [52]. Within HD patient populations there appears to be a prognostic relationship between elevated circulating e-EVs and endothelial damage $[51,60]$. Within HD patients the early loss of vascular access due to vessel stenosis and thrombosis (adverse events of HD) correlates with further elevations beyond ESRD levels of e-EVs and p-EVs [45]. Conversely, reductions in EVs are associated with reduced adverse events, as seen in a study of 40 CPB cardiac surgery patients which showed that when $\mathrm{EV}$ formation was reduced by nitric oxide and iloprost there was less thrombocytopenia and reduced post-operative bleeding [61]. Together these studies demonstrate that EVs frequently correlate with adverse outcomes in ExCor/ ECLS. Before EVs may be applied as biomarkers of ExCor/ECLS related adverse events, important questions need clarification to facilitate meaningful interpretation of the results such as teasing out the contributions specifically from patient related factors as opposed to ExCor/ECLS factors in generating the EV profiles seen, and the utility of specific EV subsets as biomarkers. A a challenging limitation of many ExCor/ECLS studies 
examining EVs is to definitively show if increases in EVs are related to simply more profound disease states within patients (leading to increased ExCor/ECLSindependent susceptibility to ExCor/ECLS-adverse events) or if increased EVs directly reflect ExCor/ECLS specific changes associated with ExCor/ECLS-adverse events? Yet, a fascinating aspect of elevated levels of EVs in patients supported by ExCor/ECLS, potentially independent of how they are formed (patient-related vs directly ExCor/ECLS-related factors), is their potential to agonize ExCor/ECLS-associated adverse events.

\section{EVS ARE MECHANISTICALLY LINKED TO ADVERSE EVENTS PERTINENT TO EXCOR/ECLS USE}

Beyond considering EVs solely as potential biomarkers of ExCor/ECLS adverse events due to their association with ExCor/ECLS and associated adverse events, it is important to note that they also have the capacity to agonize processes leading to complications (Figure 2), and as such may be targets for therapeutic intervention.

EVs can influence their environment and reprogram target cells by signalling through cell receptor interactions, delivery or stimulation of release of cytokines or transferring genetic materials such as mi/mRNAs, proteins and lipids or even dock with cells or be engulfed which can change recipient cell phenotypes and functions [17, 62, 63]. By such means, EVs can influence physiologic processes during ExCor/ECLS use via a number of factors such as increasing cell-cell adhesions with endothelial cells $[27,64]$, activating complement (by adhering to C1q, C3 and C4) [21], increasing pro-inflammatory cytokine release (such as IL-6 [64]), transporting injurious enzymes (such as myeloperoxidase [65]), presenting antigens and adhesion factors capable of leading to in vitro $\mathrm{T}$ cell

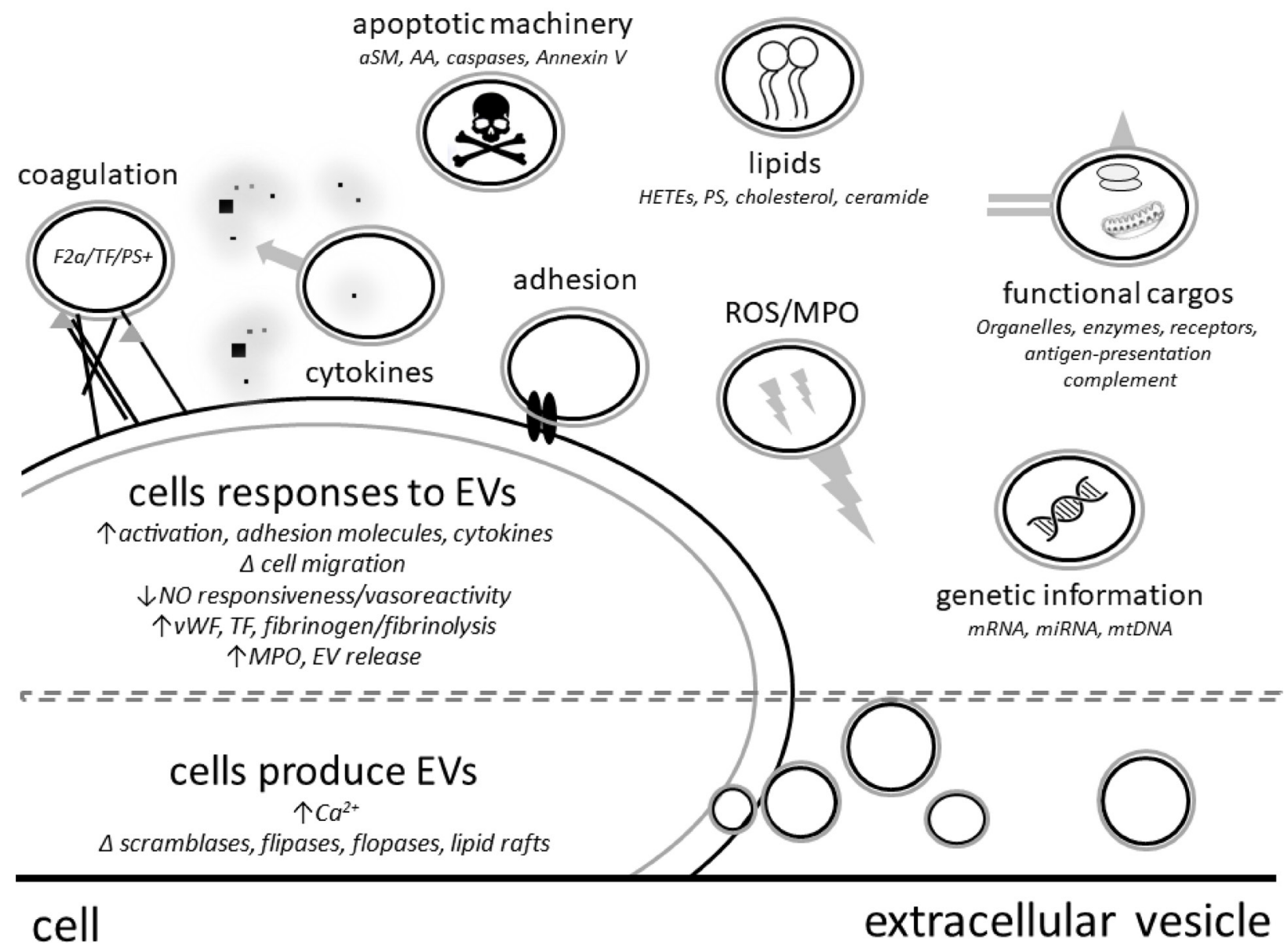

Figure 2: Extracellular vesicles (EVs) have direct or cell mediated effector functions such as release of cytokines, apoptosis mediators or reactive oxygen species (ROS). ABOVE DASHED LINE - EVs can contain functional cargos such as genetic material, organelles, enzymes (aSM: acid sphingomyelinase; MPO: myeloperoxidase), surface receptors (TF: tissue factor), coagulation factors (F2a: thrombin), apoptotic machinery and lipid components (PS: phosphatidylserine; AA: arachidonic acid; HETEs: Hydroxyeicosatetraenoic acids). BELOW DASHED LINE - EVs are formed from cells under conditions of stress or activation resulting changes such as increased intracellular calcium, altered activity of cell membrane symmetry enzymes such as scramblases, flippases and floppases. 
activation and proliferation [66] and in disease states reduce $\mathrm{EV}(\mathrm{NO})$ mediated vascular relaxation (Figure 2) [67-70]. As a specific example: under high shear stresses which resemble conditions in some ExCor/ECLS systems, p-EVs were shown to enhance the production of cytokines such as TNF $\alpha$, IL- $1 \beta$ and IL- 8 as well as adhesion molecules on leukocytes and endothelial cells which in turn enhances their interaction [27].

Thrombosis is a serious risk with ExCor/ECLS use and EVs can contribute in a number of ways to a procoagulant milieu. Endothelial damage during ExCor/ ECLS use can increase expression of VWF, fibrinogen and tissue factor (TF) on circulating e-EVs $[42,71]$. In addition, EVs from different cell types beyond endothelial cells can be adorned with TF and PS which additionally contribute negatively charged phospholipid surfaces and activation signals which assist in catalyzing the coagulation cascade. Along these lines, EVs derived from pericardial suctions have procoagulant activity as they were shown to enhance TF/FVII dependent thrombin formation in vitro [72]. Elevated levels of p- and e-EVs in HD patients have also been shown to lead to increased release of thrombin [50]. Moreover, some p-EVs have previously been shown to have potent procoagulant function described as 100-fold higher than activated platelets [73].

\section{STRATEGIES OF CONTROLLING LEVELS OF EVS IN EXCOR/ECLS}

As evidence emerges of the ability of EVs to not only act as biomarkers but additionally mediate inflammatory processes and changes within the coagulation cascade which culminate in ExCor/ECLS adverse events strategies aiming to improve the safety of ExCor/ECLS systems should focus on minimizing the negative impact of EVs during ExCor/ECLS use. This may involve removal of detrimental EVs, preventing $E V$ generation, or selection for beneficial EVS.

Specific configurations of ExCor/ECLS systems allow for clearance of detrimental EVS. In vitro experiments using continuous veno-veno hemodialysis (CVVH) circuits, hemofiltration circuits, apheresis machines and cell saver devices have shown capabilities to lower EVs by filtration [74-77]. Cell saver technology often used in combination with CPB in cardiac surgeries may in particular be an attractive means to efficiently remove $r$ - and p-EVs as shown in 13 patients who underwent $\mathrm{CPB}$, and in which the cell saver was able to remove more than $97 \%$ of EVs from ExCor/ECLS associated patient blood [75]. When e-EVs were placed in saline simulating a human's circulating volume and filtered with a standard CVVH $200 \mathrm{~nm}$ filter at flows of $250 \mathrm{ml} / \mathrm{h} 50 \%$ of Annexin $\mathrm{V}+\mathrm{e}-\mathrm{EVs}$ were cleared at 30 minutes and after 4 hours there were as few as $5.7 \%$ of circulating e-EVs remaining [74]. This may be of particular interest as PS+ EVs have been shown to be associated with ExCor/ECLS adverse events [56], so selective clearance of annexin $\mathrm{V}+\mathrm{EVs}$ may be especially beneficial. E-EVs were consistently lowered post-HD in two different studies which examined 51 HD patients $[53,78]$. A study examining HD patients showed indirect evidence of the ability of ExCor systems to clear EVs from the circulation by a net loss of prothrombotic activity post filtration, which returned after EVs were selectively returned to post-filtration plasmas [50]. The type of HD is an interesting area of future investigation concerning clearing EVs as there are differences in post-hemofiltration reductions in e-EVs in low-flux when compared with highflux HD $[60,79]$. HD with endogenous reinfusion appears to be a dialysis strategy capable of enhancing reduction of CD31+ Annexin V+e-EVs from the circulation [53]. Like HD circuits, plasma-, leuko- and platelet-pheresis (apheresis) machines are capable of lowering EVs (p- and $\mathrm{m}-\mathrm{EVs}$ ) in the circulation of patients $[76,80,81]$. Not all apheresis studies, however, consistently show changes in levels of EVs (Table 8), as other studies have shown specifically no significant differences between p-EV levels before and after filtration [82]. The authors theorized that though the apheresis circuit may have filtered many $\mathrm{p}-\mathrm{EV} \mathrm{s}$ more may have been created due to platelet activation by the circuit. Notably, however, these EV levels were measured by ELISA and not flow cytometry which offers greater sensitivity to detect differences in numbers of p-EVs. Moreover, different configurations may impact platelet activation or the ability to filter EVs, as it has been shown that different apheresis ExCor systems or use of single compared with double needle systems within a single ExCor system influence EV counts [83]. Similarly, the type of materials - including differences in pore volumes, surface area and charge density - used in absorbers in apheresis machines influences which cells adhere and EV levels [84]. Cell-adhesiveness of filters/ absorbent polymers may influence which types of cells adhere, which in turn may influence EV generation from these cells. Small pore sizes such as $75 \mathrm{~nm}$, can lead to removal of EVs, while preserving proteins, lipoproteins and coagulation factors [85]. Over and above that, not all ExCor systems may be able to efficiently filter uremic toxins, which may impact EV levels. Some uremic toxins such as indoxyl sulphate and p-cresyl sulphate are not universally cleared by ExCor systems such as $\mathrm{HD}$ and can contribute to e-EV production [46, 68]. As such Faure and coworkers noted that un-dialyzed chronic renal failure (CRF) patients had similar levels of e-EVs as dialysis patients possibly due to similar uremic burdens between these groups [46]. This concept is corroborated by the finding that in vitro stimulation of human umbilical vascular endothelial cells with uremic toxins elicits e-EV formation [46]. In rheumatoid arthritis patients leukopheresis removed p-EVs and increased antiinflammatory g-EVs, suggesting that different EV subsets 
may be individually modulated [80]. These studies provide important insights of how select ExCor/ECLS systems with specific configurations may deplete circulating EVs and may guide the way to the design of modified ExCor/ECLS systems optimized for EV removal. To this end, it is particularly advantageous that selectivity appears obtainable, as seen by filters $(75 \mathrm{~nm})$ capable of reducing prothrombogenic EVs but preserving coagulation function in plasma samples [85]; as sequestration and removal of too many of the constituents of the coagulation cascade could lead to significant coagulopathy. Further investigations are urgently needed to test the consequences and thus potential applications of EV removal in patients by filtration with ExCor/ECLS systems.

Pharmacologic agents may be able to reduce EV formation which in turn may be beneficial in reducing adverse events associated with ExCor/ECLS use. ExCor/ ECLS systems enriched with nitric oxide (20 ppm) or prostaglandins (iloprost $(2 \mathrm{ng} / \mathrm{kg} / \mathrm{min})$ ) reduce platelet activation and prevent $\mathrm{p}-\mathrm{EV}$ formation [61]. While these effects were associated with less post-operative chest tube bleeding, a direct causative link between the reduced $\mathrm{p}-\mathrm{EV}$ formation and the improved coagulation state remains to be shown [61]. Other cardiovascular medications may also have beneficial effects on circulating EV levels as there is growing evidence that angiotensin-receptor blockers and $\mathrm{HMG}-\mathrm{CoA}$ reductase inhibitors (statins) can lower p-EVs in ESRD patients [50] and m-EVs in hypertensive or diabetic patients [86]. Similarly, statins have been shown to reduce e-EV formation from TNF $\alpha$ stimulated human coronary endothelial cells in vitro [87]. During $\mathrm{CPB}$, elevations of $\mathrm{p}-\mathrm{EV}$ s can be prevented by treating platelets with GP2 $\alpha 3 \beta$ inhibitors and heparin [88], and at the experimental level, agents such as caspase-, PI3Kand $\mathrm{P}(2) \mathrm{Y}$-inhibitors further reduce platelet activation and p-EV formation $[89,90]$. Considering these medications for the goal of reducing EVs would, however, require careful attention to the specificity of their effects and potential adverse events such as bleeding or platelet imbibition. A future area of investigation may include a means of mitigating the systemic effects of these medications with strategies such as having them coated on ExCor/ ECLS surfaces instead of systemic administration into the patient's circulation. Preliminary tissue engineering experiments involving lining oxygenator membranes under static conditions with fibronectin have shown preferential adherence of circulating endothelial cells [91]. Lining circuits with endothelial cells may help reduce foreign material exposure to blood and lower EV formation due to decreased cellular activation and inflammation in patients requiring long term ExCor/ECLS support such as those having destination therapy VADs. At the same time, however, an endothelial cell lining on ExCor/ECLS systems may become a relevant source of e-EV production due to high shear forces generated by blood circulating within ExCor/ECLS systems. Beyond lining circuits with fibronectin or heparin, it may be possible to line sections of extracorporeal circuits with immobilized antibodies (such as IgM [92]) or other adhesive molecules to selectively sequester and eliminate EVs within patients connected to ExCor/ECLS systems. Beyond parenteral or enteral route medications, oxygen tension may impact EV numbers in patients with ExCor/ECLS systems. Hyperoxia has the potential for EV formation in part due to neutrophil and platelet activation, aggregation and reactive oxygen species (ROS) production [93, 94] and vice versa hypoxia is a known trigger for EV formation [95]. Strict regulation of partial pressures of oxygen may thus prove an accessible strategy to reduce EVs. Taken together, existing and experimental drugs, bioengineering approaches or revised SOPs for ExCor/ECLS patient care such as adhesion to normoxic ventilation may offer potential means of reducing circulating EVs passing through ExCor/ECLS systems.

Lastly, not all EVs are detrimental, and as such should not necessarily be removed from the circulation. Of the EV populations that may have therapeutic potential, endothelial progenitor cell (EPC)-EVs and mesenchymal stem cell (MSC)-EVs show presently the most promise. EPC-EVs improve ischemic renal injury [96] which is a complication relevant to ExCor/ECLS use. Erythropoietin treatment in ESRD patients dose dependently boosts MSC-EV formation which proved beneficial in animal models of renal injury [97]. Beneficial effects may, however, not be limited to stem- or progenitor-cell derived EVs. Though e-EVs from diseased patients can do harm as outlined in previous sections of this review, e-EVs from healthy individuals can transport eNOS which may be able to boost intravascular NO generation [70]. Under normal/healthy conditions e-EVs carry functional eNOS, whereas during states of disease, e-EV eNOS expression is lost which leads to less NO formation which is a prorelaxant mediator for vascular/endothelial tone. As such selective delivery of healthy e-EVs may be beneficial to certain patients with ExCor/ECLS systems. In order for EVs to become a cell-free therapeutic option, more studies are needed to decipher which EVs may broker benefits as opposed to harms, and strategies are required to concentrate beneficial EVs while removing harmful EVs.

\section{TECHNICAL CHALLENGES OF USING EVS AS BIOMARKERS OF EXCOR/ECLS ADVERSE EVENTS}

As the study of EVs as biomarkers and effectors of disease evolves, hurdles remain such as limitations in strategies for their isolation and accurate characterization. Challenges associated with reproducible collection and analysis of EVs are summarized in a recent review [98]. EVs exist in many biological fluids. The most popular site for monitoring EVs pertinent to ExCor/ECLS use is blood. In order to optimally measure EVs in anticoagulated blood samples, it is recommended to rapidly ( $<1$ hour) 
remove cells and debris by differential centrifugation, which may lead to confounding false positive counting of non-EVs such as seen with small platelets, or even lead to post-collection generation of EVs if cells activate during in vitro processing [99]. In most ExCor/ECLS studies, EVs are measured by flow cytometry which offers advantages of being accessible and allowing high throughput analysis of many EVs. Alternative high throughput EV analysis tools exist such as Nanotracker technology, mass spectroscopy and tunable resistive pulse sensing. Other complimentary techniques for EV characterization comprise functional assays to assess EV surface receptors or procoagulant capacity using enzyme linked immunosorbent assays or single EV analyses with electron and confocal laser microscopy. Interpretation of EV studies can be complicated, though, as analytical tools for EV detection such as flow cytometers (the most popular detection device for ExCor/ECLS EV studies) function at their lower limit of detection to measure small EVs which can be confounded by instrument noise or debris, while at the upper size limit measurements can be complicated by the presence of platelets or EV-platelet aggregates. Beyond aggregation which can lead to inaccurate detection of EVs, fusion or adherence of MPs to other EVs or cells has been reported in ExCor/ECLS EV studies which add further challenges to enumerating $\operatorname{EVs}[3,37,48,100]$. Taken together, while use of EVs as biomarkers is rapidly evolving, the basic methodology for their standardized assessment is still under development. Conventions and guidelines for acquisition, handling and interpretation of EVs from ExCor/ECLS studies are needed.

\section{UNKNOWNS AND FUTURE DIRECTIONS}

Beyond the ExCor/ECLS configurations discussed in this review, there are others that have not yet been assessed in terms of their effects on EV formation and characteristics, such as small circulatory assist devices like the impala or ex vivo solid organ transplant perfusion circuits. An emerging example of novel ExCor/ECLS systems are experimental or clinical ex vivo organ perfusion systems for lung, heart, kidney and liver [101-104] which remain largely unstudied. Beyond different types of circuits, this review did not address the possible presence and contribution of exosomes and apoptotic bodies, which may be important in terms of biomarkers or pathomechanistic effectors. Yet there is presently a paucity of publications examining these EVs in the context of ExCor/ECLS use which thus presents an important area for future investigation. An exciting aspect of research is assessing the impact of EVs seen in ExCor/ECLS circuits in terms of their capacity to mediate disease processes and accordingly, the implementation of EV targeted strategies in ExCor/ECLS systems to alleviate these detrimental adverse effects.

\section{CONCLUSIONS}

Most ExCor/ECLS systems (acute- or prolongedsupport) lead to transient or permanently increased levels of circulating EVs. This had fueled the idea that they may be exploited as biomarkers for prognosis or indicators of potential ExCor/ECLS-related adverse effects; but recently also generated interest in the role of EVs as propagators of disease processes and mechanisms. Within ExCor/ECLS circuits certain components such as oxygenators regionally agonize EV formation. Levels of circulating EVs increase primarily during ExCor/ECLS use, and return to lower levels with discontinuation of circuit use. When characterizing the contribution of ExCor/ECLS systems to elevated EV levels from patients, it is, however, important to consider the basal levels of EVs which may be elevated prior to ExCor/ECLS exposure due to underlying diseases and comorbidities. EVs offer potential as biomarkers to predict adverse events associated with ExCor/ECLS use, and monitoring abundance, time profiles, and antigenicity of EVs has potential to become implemented as routine screening and monitoring tool into clinical practice. The most numerous EVs in circulation appear to be $\mathrm{p}-\mathrm{EV}$ s. The presence of $\mathrm{PS}+$, p- or e-EVs each can correlate to adverse events related to use of different ExCor/ECLS systems.

New insights and opportunities are emerging which may improve care for patients requiring ExCor/ECLS use. Though many ExCor/ECLS systems appear to agonize EV production it seems select ExCor/ECLS sytems may offer capacity to reduce their levels. Manipulation of EV numbers or subtypes may be of use for reducing disease and improving coagulation status in the context of anticoagulated patients paired with ExCor/ECLS. Future ExCor/ECLS designs may involve optimization for controlling EV levels and subtypes. These opportunities are not without risk. EVs, as described above can have both beneficial and detrimental effects in biology. The consequences of indiscriminate or selective reduction/ removal of EVs or their manipulation is not well understood at present, in terms of physiological changes.

Manipulation of EVs may be of particular interest in oncology, where they are associated with dissemination of metastatic disease [19]. Cancer cell EVs can lead to immunomodulation and promote tumor growth and metastasis, therefore developing strategies such as ExCor/ ECLS systems to remove unwanted EVs could be an attractive adjunct strategy when treating cancer patients $[19,77]$. Though typically, advanced metastatic disease is a contraindication to ExCor/ECLS use, employing ExCor/ ECLS to reduce EVs may allow reduced progression of metastases and prolong quality of life. Boosting beneficial EV subpopulations such as EPC- or MSC-EVs may be an even more ambitious, but ultimately rewarding strategy with the aim to establish beneficial EVs as cell-free cell therapeutics the use of which would not be limited to ExCor/ECLS. 


\section{CONFLICTS OF INTEREST}

The authors declare no competing interests.

\section{FUNDING}

Support was provided from institutional and departmental sources. MJM was funded by a Canadian Institute of Health Research Vanier Scholarship

\section{REFERENCES}

1. Wohlfarth P, Ullrich R, Staudinger T, Bojic A, Robak O, Hermann A, Lubsczyk B, Worel N, Fuhrmann V, Schoder M, Funovics M, Rabitsch W, Knoebl P, et al. Extracorporeal membrane oxygenation in adult patients with hematologic malignancies and severe acute respiratory failure. Crit Care. 2014; 18:R20. https://doi.org/10.1186/cc13701.

2. Rosskopfova P, Perentes JY, Ris HB, Gronchi F, Krueger T, Gonzalez M. Extracorporeal support for pulmonary resection: current indications and results. World J Surg Oncol. 2016; 14:25. https://doi.org/10.1186/ s12957-016-0781-0.

3. Chan CH, Pieper IL, Hambly R, Radley G, Jones A, Friedmann Y, Hawkins KM, Westaby S, Foster G, Thornton CA. The CentriMag Centrifugal Blood Pump as a Benchmark for In Vitro Testing of Hemocompatibility in Implantable Ventricular Assist Devices. Artif Organs. 2015; 39:93-101. https://doi.org/10.1111/aor.12351.

4. Ivak P, Pitha J, Netuka I. Circulating microparticles as a predictor of vascular properties in patients on mechanical circulatory support; hype or hope? Physiol Res. 2016. 65:727-735

5. Schwartz J, Winters JL, Padmanabhan A, Balogun RA, Delaney M, Linenberger ML, Szczepiorkowski ZM, Williams ME, Wu Y, Shaz BH. Guidelines on the use of therapeutic apheresis in clinical practice-evidence-based approach from the Writing Committee of the American Society for Apheresis: the sixth special issue. J Clin Apher. 2013; 28:145-284. https://doi.org/10.1002/jca.21276.

6. Leligdowicz A, Fan E. Extracorporeal life support for severe acute respiratory distress syndrome. Curr Opin Crit Care. 2015; 21:13-9. https://doi.org/10.1097/ mcc.0000000000000170.

7. Demiselle J, Besson V, Sayegh J, Subra JF, Augusto JF. Total Artificial Heart and Chronic Haemodialysis: A Possible Bridge to Transplantation. Blood Purif. 2016; 42:301-3. https://doi.org/10.1159/000448162.

8. Birks EJ. A changing trend toward destination therapy: are we treating the same patients differently? Tex Heart Inst J. 2011; 38:552-4.

9. Sauer CM, Yuh DD, Bonde P. Extracorporeal membrane oxygenation use has increased by $433 \%$ in adults in the
United States from 2006 to 2011. ASAIO J. 2015; 61:31-6. https://doi.org/10.1097/mat.0000000000000160.

10. McCarthy FH, McDermott KM, Kini V, Gutsche JT, Wald JW, Xie D, Szeto WY, Bermudez CA, Atluri P, Acker MA, Desai ND. Trends in U.S. Extracorporeal Membrane Oxygenation Use and Outcomes:2002-2012. Semin Thorac Cardiovasc Surg. 2015; 27:81-8. https://doi.org/10.1053/j. semtcvs.2015.07.005.

11. Cove ME, MacLaren G, Federspiel WJ, Kellum JA. Bench to bedside review: Extracorporeal carbon dioxide removal, past present and future. Crit Care. 2012; 16:232. https://doi. org/10.1186/cc11356.

12. Del Sorbo L, Cypel M, Fan E. Extracorporeal life support for adults with severe acute respiratory failure. Lancet Respir Med. 2014; 2:154-64. https://doi.org/10.1016/ s2213-2600(13)70197-8.

13. Karagiannidis C, Brodie D, Strassmann S, Stoelben E, Philipp A, Bein T, Muller T, Windisch W. Extracorporeal membrane oxygenation: evolving epidemiology and mortality. Intensive Care Med. 2016; 42:889-96. https:// doi.org/10.1007/s00134-016-4273-z.

14. Venema LH, Sharma AS, Simons AP, Bekers O, Weerwind PW. Contemporary Oxygenator Design Relative to Hemolysis. J Extra Corpor Technol. 2014; 46:212-6.

15. Wurzinger LJ, Opitz R, Blasberg P, Schmid-Schonbein H. Platelet and coagulation parameters following millisecond exposure to laminar shear stress. Thromb Haemost. 1985; 54:381-6.

16. Buzas EI, Gyorgy B, Nagy G, Falus A, Gay S. Emerging role of extracellular vesicles in inflammatory diseases. Nat Rev Rheumatol. 2014; 10:356-64. https://doi.org/10.1038/ nrrheum.2014.19.

17. McVey M, Tabuchi A, Kuebler WM. Microparticles and acute lung injury. Am J Physiol Lung Cell Mol Physiol. 2012; 303:L364-81. https://doi.org/10.1152/ ajplung.00354.2011.

18. Lancaster GI, Febbraio MA. Exosome-dependent trafficking of HSP70: a novel secretory pathway for cellular stress proteins. J Biol Chem. 2005; 280:23349-55. https://doi. org/10.1074/jbc.M502017200.

19. Becker A, Thakur BK, Weiss JM, Kim HS, Peinado H, Lyden D. Extracellular Vesicles in Cancer: Cell-to-Cell Mediators of Metastasis. Cancer Cell. 2016; 30:836-48. https://doi.org/10.1016/j.ccell.2016.10.009.

20. Fabre O, Vincentelli A, Corseaux D, Juthier F, Susen S, Bauters A, Van Belle E, Mouquet F, Le Tourneau T, Decoene C, Crepin F, Prat A, Jude B. Comparison of blood activation in the wound, active vent, and cardiopulmonary bypass circuit. Ann Thorac Surg. 2008; 86:537-41. https:// doi.org/10.1016/j.athoracsur.2008.02.076.

21. Biro E, van den Goor JM, de Mol BA, Schaap MC, Ko LY, Sturk A, Hack CE, Nieuwland R. Complement activation on the surface of cell-derived microparticles during cardiac surgery with cardiopulmonary bypass - is retransfusion 
of pericardial blood harmful? Perfusion. 2011; 26:21-9. https://doi.org/10.1177/0267659110385742.

22. Ding J, Chen Z, Niu S, Zhang J, Mondal NK, Griffith BP, Wu ZJ. Quantification of Shear-Induced Platelet Activation: High Shear Stresses for Short Exposure Time. Artif Organs. 2015; 39:576-83. https://doi.org/10.1111/aor.12438.

23. Dewanjee MK, Wu SM, Kapadvanjwala M, De D, Dewanjee S, Gonzalez L, Novak S, Hsu LC, Perryman RA, Duncan RC, Serafini AN, Sfakianakis GN, Horton AF. Emboli from an extraluminal blood flow hollow fiber oxygenator with and without an arterial filter during cardiopulmonary bypass in a pig model. ASAIO J. 1996; 42:1010-8.

24. Meyer AD, Gelfond JA, Wiles AA, Freishtat RJ, RaisBahrami K. Platelet-derived microparticles generated by neonatal extracorporeal membrane oxygenation systems. ASAIO J. 2015; 61:37-42. https://doi.org/10.1097/ mat.0000000000000164.

25. Eckman PM, John R. Bleeding and thrombosis in patients with continuous-flow ventricular assist devices. Circulation. 2012; 125:3038-47. https://doi.org/10.1161/ circulationaha.111.040246.

26. Brown CH 3rd, Leverett LB, Lewis CW, Alfrey CP Jr., Hellums JD. Morphological, biochemical, and functional changes in human platelets subjected to shear stress. J Lab Clin Med. 1975; 86:462-71.

27. Nomura S, Tandon NN, Nakamura T, Cone J, Fukuhara $\mathrm{S}$, Kambayashi J. High-shear-stress-induced activation of platelets and microparticles enhances expression of cell adhesion molecules in THP-1 and endothelial cells. Atherosclerosis. 2001; 158:277-87.

28. Idriss NK, Blann AD, Sayed DM, Gaber MA, Hassen HA, Kishk YT. Circulating Endothelial Cells and Platelet Microparticles in Mitral Valve Disease With and Without Atrial Fibrillation. Angiology. 2015; 66:631-7. https://doi. org/10.1177/0003319714546183.

29. Jenkins NT, Padilla J, Boyle LJ, Credeur DP, Laughlin MH, Fadel PJ. Disturbed blood flow acutely induces activation and apoptosis of the human vascular endothelium. Hypertension. 2013; 61:615-21. https://doi.org/10.1161/ hypertensionaha.111.00561.

30. Rhodes NP, Shortland AP, Rattray A, Black RA, Williams DF. Activation status of platelet aggregates and platelet microparticles shed in sheared whole blood. J Mater Sci Mater Med. 1997; 8:747-51.

31. Rand ML, Wang H, Bang KW, Packham MA, Freedman J. Rapid clearance of procoagulant plateletderived microparticles from the circulation of rabbits. J Thromb Haemost. 2006; 4:1621-3. https://doi. org/10.1111/j.1538-7836.2006.02011.x.

32. Owens AP 3rd, Mackman N. Microparticles in hemostasis and thrombosis. Circ Res. 2011; 108:1284-97. https://doi. org/10.1161/CIRCRESAHA.110.233056.

33. Rank A, Nieuwland R, Crispin A, Grutzner S, Iberer M, Toth B, Pihusch R. Clearance of platelet microparticles in vivo. Platelets. 2011; 22:111-6. https://doi.org/10.3109/0 9537104.2010.520373.

34. Miyamoto S, Marcinkiewicz C, Edmunds LH Jr, Niewiarowski S. Measurement of platelet microparticles during cardiopulmonary bypass by means of captured ELISA for GPIIb/IIIa. Thromb Haemost. 1998; 80:225-30.

35. Augustine D, Ayers LV, Lima E, Newton L, Lewandowski AJ, Davis EF, Ferry B, Leeson P. Dynamic release and clearance of circulating microparticles during cardiac stress. Circ Res. 2014; 114:109-13. https://doi.org/10.1161/ circresaha.114.301904.

36. van den Goor JM, van den Brink A, Nieuwland R, van Oeveren W, Rutten PM, Tepaske R, Tijssen JG, Sturk A, de Mol BA, Eijsman L. Generation of platelet-derived microparticles in patients undergoing cardiac surgery is not affected by complement activation. J Thorac Cardiovasc Surg. 2003; 126:1101-6.

37. Chan CH, Hilton A, Foster G, Hawkins KM, Badiei N, Thornton CA. The evaluation of leukocytes in response to the in vitro testing of ventricular assist devices. Artif Organs. 2013; 37:793-801. https://doi.org/10.1111/aor.12161.

38. Sansone R, Stanske B, Keymel S, Schuler D, Horn P, Saeed D, Boeken U, Westenfeld R, Lichtenberg A, Kelm M, Heiss C. Macrovascular and microvascular function after implantation of left ventricular assist devices in endstage heart failure: Role of microparticles. J Heart Lung Transplant. 2015; 34:921-32. https://doi.org/10.1016/j. healun.2015.03.004.

39. Pitha J, Dorazilova Z, Melenovsky V, Kralova Lesna I, Stavek P, Stepankova J, Urban M, Maly J, Netuka I. The impact of left ventricle assist device on circulating endothelial microparticles - pilot study. Neuro Endocrinol Lett. 2012 (Suppl 33); 2:68-72.

40. Ivak P, Pitha J, Wohlfahrt P, Kralova Lesna I, Stavek P, Dorazilova Z, Stepankova J, Maly J, Pokorny M, Netuka I. Endothelial dysfunction expressed as endothelial microparticles in patients with end-stage heart failure. Physiol Res. 2014 (Suppl 3); 63:S369-73.

41. Ivak P, Pitha J, Wohlfahrt P, Kralova Lesna I, Stavek P, Melenovsky V, Dorazilova Z, Hegarova M, Stepankova J, Maly J, Sekerkova A, Turcani D, Netuka I. Biphasic response in number of stem cells and endothelial progenitor cells after left ventricular assist device implantation: A 6month follow-up. Int J Cardiol. 2016; 218:98-103. https:// doi.org/10.1016/j.ijcard.2016.05.063.

42. Diehl P, Aleker M, Helbing T, Sossong V, Beyersdorf F, Olschewski M, Bode C, Moser M. Enhanced microparticles in ventricular assist device patients predict platelet, leukocyte and endothelial cell activation. Interact Cardiovasc Thorac Surg. 2010; 11:133-7. https://doi. org/10.1510/icvts.2010.232603.

43. Muthiah K, Connor D, Ly K, Gardiner EE, Andrews RK, Qiao J, Rutgers D, Robson D, Low J, Jarvis S, Macdonald P, Dhital K, Jansz P, et al. Longitudinal changes in hemostatic parameters and reduced pulsatility contribute 
to non-surgical bleeding in patients with centrifugal continuous-flow left ventricular assist devices. J Heart Lung Transplant. 2016; 35:743-51. https://doi.org/10.1016/j. healun.2015.12.024.

44. Birschmann I, Dittrich M, Eller T, Wiegmann B, Reininger AJ, Budde U, Struber M. Ambient hemolysis and activation of coagulation is different between HeartMate II and HeartWare left ventricular assist devices. J Heart Lung Transplant. 2014; 33:80-7. https://doi.org/10.1016/j. healun.2013.11.010.

45. Ryu JH, Lim SY, Ryu DR, Kang DH, Choi KB, Kim SJ. Association between vascular access failure and microparticles in hemodialysis patients. Kidney Res Clin Pract. 2012; 31:38-47. https://doi.org/10.1016/j. krcp.2011.12.002.

46. Faure V, Dou L, Sabatier F, Cerini C, Sampol J, Berland Y, Brunet P, Dignat-George F. Elevation of circulating endothelial microparticles in patients with chronic renal failure. J Thromb Haemost. 2006; 4:566-73. https://doi. org/10.1111/j.1538-7836.2005.01780.x.

47. Jourde-Chiche N, Dou L, Sabatier F, Calaf R, Cerini C, Robert S, Camoin-Jau L, Charpiot P, Argiles A, Dignat-George F, Brunet P. Levels of circulating endothelial progenitor cells are related to uremic toxins and vascular injury in hemodialysis patients. J Thromb Haemost. 2009; 7:1576-84. https://doi.org/10.1111/j.1538-7836.2009.03540.x.

48. Daniel L, Fakhouri F, Joly D, Mouthon L, Nusbaum P, Grunfeld JP, Schifferli J, Guillevin L, Lesavre P, Halbwachs-Mecarelli L. Increase of circulating neutrophil and platelet microparticles during acute vasculitis and hemodialysis. Kidney Int. 2006; 69:1416-23. https://doi. org/10.1038/sj.ki.5000306.

49. Trappenburg MC, van Schilfgaarde M, Frerichs FC, Spronk HM, ten Cate H, de Fijter CW, Terpstra WE, Leyte A. Chronic renal failure is accompanied by endothelial activation and a large increase in microparticle numbers with reduced procoagulant capacity. Nephrol Dial Transplant. 2012; 27:1446-53. https://doi.org/10.1093/ndt/gfr474.

50. Burton JO, Hamali HA, Singh R, Abbasian N, Parsons R, Patel AK, Goodall AH, Brunskill NJ. Elevated levels of procoagulant plasma microvesicles in dialysis patients. PLoS One. 2013; 8:e72663. https://doi.org/10.1371/journal. pone. 0072663 .

51. Merino A, Portoles J, Selgas R, Ojeda R, Buendia P, Ocana J, Bajo MA, del Peso G, Carracedo J, Ramirez R, MartinMalo A, Aljama P. Effect of different dialysis modalities on microinflammatory status and endothelial damage. Clin J Am Soc Nephrol. 2010; 5:227-34. https://doi.org/10.2215/ cjn.03260509.

52. Dursun I, Poyrazoglu HM, Gunduz Z, Ulger H, Yykylmaz A, Dusunsel R, Patyroglu T, Gurgoze M. The relationship between circulating endothelial microparticles and arterial stiffness and atherosclerosis in children with chronic kidney disease. Nephrol Dial Transplant. 2009; 24:2511-8. https:// doi.org/10.1093/ndt/gfp066.
53. Esquivias-Motta E, Martin-Malo A, Buendia P, AlvarezLara MA, Soriano S, Crespo R, Carracedo J, Ramirez R, Aljama P. Hemodiafiltration With Endogenous Reinfusion Improved Microinflammation and Endothelial Damage Compared With Online-Hemodiafiltration: A Hypothesis Generating Study. Artif Organs. 2016; 41:88-98. https://doi. org/10.1111/aor.12704.

54. Feng B, Chen Y, Luo Y, Chen M, Li X, Ni Y. Circulating level of microparticles and their correlation with arterial elasticity and endothelium-dependent dilation in patients with type 2 diabetes mellitus. Atherosclerosis. 2010; 208:264-9. https:// doi.org/10.1016/j.atherosclerosis.2009.06.037.

55. Amabile N, Guerin AP, Tedgui A, Boulanger CM, London GM. Predictive value of circulating endothelial microparticles for cardiovascular mortality in end-stage renal failure: a pilot study. Nephrol Dial Transplant. 2012; 27:1873-80. https://doi.org/10.1093/ndt/gfr573.

56. Nascimbene A, Hernandez R, George JK, Parker A, Bergeron AL, Pradhan S, Vijayan KV, Civitello A, Simpson L, Nawrot M, Lee VV, Mallidi HR, Delgado RM, et al. Association between cell-derived microparticles and adverse events in patients with nonpulsatile left ventricular assist devices. J Heart Lung Transplant. 2014; 33:470-7. https://doi.org/10.1016/j.healun.2014.01.004.

57. Shah KB, Kontos MC. Microparticles and left ventricular assist device complications: a causal association? J Heart Lung Transplant. 2014; 33:468-9. https://doi.org/10.1016/j. healun.2014.02.031.

58. Lekakis J, Abraham P, Balbarini A, Blann A, Boulanger CM, Cockcroft J, Cosentino F, Deanfield J, Gallino A, Ikonomidis I, Kremastinos D, Landmesser U, Protogerou A, et al. Methods for evaluating endothelial function: a position statement from the European Society of Cardiology Working Group on Peripheral Circulation. Eur J Cardiovasc Prev Rehabil. 2011; 18:775-89. https://doi. org/10.1177/1741826711398179.

59. Wittwer T, Choi YH, Neef K, Schink M, Sabashnikov A, Wahlers T. Off-pump or minimized on-pump coronary surgery-initial experience with Circulating Endothelial Cells (CEC) as a supersensitive marker of tissue damage. J Cardiothorac Surg. 2011; 6:142. https://doi. org/10.1186/1749-8090-6-142.

60. Ramirez R, Carracedo J, Merino A, Nogueras S, AlvarezLara MA, Rodriguez M, Martin-Malo A, Tetta C, Aljama P. Microinflammation induces endothelial damage in hemodialysis patients: the role of convective transport. Kidney Int. 2007; 72:108-13. https://doi.org/10.1038/sj.ki.5002250.

61. Chung A, Wildhirt SM, Wang S, Koshal A, Radomski MW. Combined administration of nitric oxide gas and iloprost during cardiopulmonary bypass reduces platelet dysfunction: a pilot clinical study. J Thorac Cardiovasc Surg. 2005; 129:782-90. https://doi.org/10.1016/j.jtcvs.2004.06.049.

62. Mulcahy LA, Pink RC, Carter DR. Routes and mechanisms of extracellular vesicle uptake. J Extracell Vesicles. 2014; 3. https://doi.org/10.3402/jev.v3.24641. 
63. Trzepizur W, Martinez MC, Priou P, Andriantsitohaina R, Gagnadoux F. Microparticles and vascular dysfunction in obstructive sleep apnoea. Eur Respir J. 2014; 44:207-16. https://doi.org/10.1183/09031936.00197413.

64. Mesri M, Altieri DC. Endothelial cell activation by leukocyte microparticles. J Immunol. 1998; 161:4382-7.

65. Pitanga TN, de Aragao Franca L, Rocha VC, Meirelles T, Borges VM, Goncalves MS, Pontes-de-Carvalho LC, Noronha-Dutra AA, dos-Santos WL. Neutrophil-derived microparticles induce myeloperoxidase-mediated damage of vascular endothelial cells. BMC Cell Biol. 2014; 15:21. https://doi.org/10.1186/1471-2121-15-21.

66. Wheway J, Latham SL, Combes V, Grau GE. Endothelial microparticles interact with and support the proliferation of T cells. J Immunol. 2014; 193:3378-87. https://doi. org/10.4049/jimmunol.1303431.

67. Amabile N, Guerin AP, Leroyer A, Mallat Z, Nguyen C, Boddaert J, London GM, Tedgui A, Boulanger CM. Circulating endothelial microparticles are associated with vascular dysfunction in patients with end-stage renal failure. J Am Soc Nephrol. 2005; 16:3381-8. https://doi. org/10.1681/asn.2005050535.

68. Jourde-Chiche N, Dou L, Cerini C, Dignat-George $\mathrm{F}$, Brunet P. Vascular incompetence in dialysis patients - protein-bound uremic toxins and endothelial dysfunction. Semin Dial. 2011; 24:327-37. https://doi. org/10.1111/j.1525-139X.2011.00925.x.

69. Brodsky SV, Zhang F, Nasjletti A, Goligorsky MS. Endothelium-derived microparticles impair endothelial function in vitro. Am J Physiol Heart Circ Physiol. 2004; 286: H1910-5. https://doi.org/10.1152/ajpheart.01172.2003.

70. Horn P, Cortese-Krott MM, Amabile N, Hundsdorfer C, Kroncke KD, Kelm M, Heiss C. Circulating microparticles carry a functional endothelial nitric oxide synthase that is decreased in patients with endothelial dysfunction. J Am Heart Assoc. 2013; 2:e003764. https://doi.org/10.1161/ jaha.112.003764.

71. Rabelink TJ, de Boer HC, van Zonneveld AJ. Endothelial activation and circulating markers of endothelial activation in kidney disease. Nat Rev Nephrol. 2010; 6:404-14. https://doi.org/10.1038/nrneph.2010.65.

72. Nieuwland R, Berckmans RJ, Rotteveel-Eijkman RC, Maquelin KN, Roozendaal KJ, Jansen PG, ten Have K, Eijsman L, Hack CE, Sturk A. Cell-derived microparticles generated in patients during cardiopulmonary bypass are highly procoagulant. Circulation. 1997; 96:3534-41.

73. Sinauridze EI, Kireev DA, Popenko NY, Pichugin AV, Panteleev MA, Krymskaya OV, Ataullakhanov FI. Platelet microparticle membranes have 50- to 100 -fold higher specific procoagulant activity than activated platelets. Thromb Haemost. 2007; 97:425-34.

74. Abdelhafeez AH, Jeziorczak PM, Schaid TR, Hoefs SL, Kaul S, Nanchal R, Jacobs ER, Densmore JC. Clinical CVVH model removes endothelium-derived microparticles from circulation. J Extracell Vesicles. 2014; 3. https://doi. org/10.3402/jev.v3.23498.
75. van den Goor JM, Nieuwland R, van Oeveren W, Rutten PM, Tijssen JG, Hau CM, Sturk A, Eijsman L, de Mol BA. Cell Saver device efficiently removes cell-derived microparticles during cardiac surgery. J Thorac Cardiovasc Surg. 2007; 134:798-9. https://doi.org/10.1016/j. jtcvs.2007.02.042.

76. Connolly KD, Willis GR, Datta DB, Ellins EA, Ladell K, Price DA, Guschina IA, Rees DA, James PE. Lipoproteinapheresis reduces circulating microparticles in individuals with familial hypercholesterolemia. J Lipid Res. 2014; 55:2064-72. https://doi.org/10.1194/jlr.M049726.

77. Marleau AM, Chen CS, Joyce JA, Tullis RH. Exosome removal as a therapeutic adjuvant in cancer. J Transl Med. 2012; 10:134. https://doi.org/10.1186/1479-5876-10-134.

78. Boulanger CM, Amabile N, Guerin AP, Pannier B, Leroyer AS, Mallat CN, Tedgui A, London GM. In vivo shear stress determines circulating levels of endothelial microparticles in end-stage renal disease. Hypertension. 2007; 49:902-8. https://doi.org/10.1161/01.HYP.0000259667.22309.df.

79. Ramirez R, Martin-Malo A, Aljama P. Inflammation and hemodiafiltration. Contrib Nephrol. 2007; 158:210-5. https://doi.org/10.1159/000107252.

80. Umekita K, Hidaka T, Ueno S, Takajo I, Kai Y, Nagatomo Y, Sawaguchi A, Suganuma T, Okayama A. Leukocytapheresis (LCAP) decreases the level of platelet-derived microparticles (MPs) and increases the level of granulocytes-derived MPs: a possible connection with the effect of LCAP on rheumatoid arthritis. Mod Rheumatol. 2009; 19:265-72. https://doi.org/10.1007/ s10165-009-0164-2.

81. Asai $\mathrm{T}$, Nomura $\mathrm{S}$, Ishii $\mathrm{K}$, Ichikawa $\mathrm{K}$, Umei $\mathrm{N}$, Matsunami K, Matsuo Y, Kitazawa Y. Therapeutic efficacy of leukocytapheresis for procoagulant microparticles during hemophagocytic syndrome. Pathophysiol Haemost Thromb. 2008; 36:98-101. https://doi.org/10.1159/000173729.

82. Hanafusa N, Satonaka H, Doi K, Yatomi Y, Noiri E, Fujita T. Platelet-derived microparticles are removed by a membrane plasma separator. ASAIO J. 2010; 56:323-5.

83. Black A, Pienimaeki-Roemer A, Kenyon O, Orso E, Schmitz G. Platelet-derived extracellular vesicles in plateletpheresis concentrates as a quality control approach. Transfusion. 2015; 55:2184-96. https://doi.org/10.1111/ trf. 13128 .

84. Weiss R, Eichhorn T, Spittler A, Micusik M, Fischer $\mathrm{MB}$, Weber V. Release and cellular origin of extracellular vesicles during circulation of whole blood over adsorbent polymers for lipid apheresis. J Biomed Mater Res B Appl Biomater. 2015; 105:636-646. https://doi.org/10.1002/ jbm.b.33588.

85. Chou ML, Lin LT, Devos D, Burnouf T. Nanofiltration to remove microparticles and decrease the thrombogenicity of plasma: in vitro feasibility assessment. Transfusion. 2015; 55:2433-44. https://doi.org/10.1111/trf.13162.

86. Nomura S, Shouzu A, Omoto S, Nishikawa M, Iwasaka T. Effects of losartan and simvastatin on monocyte-derived 
microparticles in hypertensive patients with and without type 2 diabetes mellitus. Clin Appl Thromb Hemost. 2004; 10:133-41.

87. Tramontano AF, O'Leary J, Black AD, Muniyappa R, Cutaia MV, El-Sherif N. Statin decreases endothelial microparticle release from human coronary artery endothelial cells: implication for the Rho-kinase pathway. Biochem Biophys Res Commun. 2004; 320:34-8. https://doi.org/10.1016/j. bbrc.2004.05.127.

88. Kondo N, Wakayama F, Suzuki Y, Fukui K, Takaya S, Fukuda I. The state of platelets preserved in extracorporeal circulation with a glycoprotein IIb/IIIa inhibitor. Thromb Res. 2004; 113:303-10. https://doi.org/10.1016/j.thromres.2004.03.017.

89. Piguet PF, Vesin C. Modulation of platelet caspases and lifespan by anti-platelet antibodies in mice. Eur J Haematol. 2002; 68:253-61.

90. Krajewski S, Kurz J, Geisler T, Peter K, Wendel HP, Straub A. Combined blockade of ADP receptors and PI3-kinase p110beta fully prevents platelet and leukocyte activation during hypothermic extracorporeal circulation. PLoS One. 2012; 7:e38455. https://doi.org/10.1371/journal. pone. 0038455 .

91. Cornelissen CG, Dietrich M, Gromann K, Frese J, Krueger S, Sachweh JS, Jockenhoevel S. Fibronectin coating of oxygenator membranes enhances endothelial cell attachment. Biomed Eng Online. 2013; 12:7. https://doi. org/10.1186/1475-925x-12-7.

92. Litvack ML, Post M, Palaniyar N. IgM promotes the clearance of small particles and apoptotic microparticles by macrophages. PLoS One. 2011; 6:e17223. https://doi. org/10.1371/journal.pone.0017223.

93. Hayes RA, Shekar K, Fraser JF. Is hyperoxaemia helping or hurting patients during extracorporeal membrane oxygenation? Review of a complex problem. Perfusion. 2013; 28:184-93. https://doi.org/10.1177/0267659112473172.

94. Brubakk AO, Ross JA, Thom SR. Saturation diving; physiology and pathophysiology. Compr Physiol. 2014; 4:1229-72. https://doi.org/10.1002/cphy.c130048.

95. Lichtenauer M, Goebel B, Fritzenwanger M, Forster M, Betge S, Lauten A, Figulla HR, Jung C. Simulated temporary hypoxia triggers the release of CD31+/Annexin+ endothelial microparticles: A prospective pilot study in humans. Clin Hemorheol Microcirc. 2014; 61:83-90. https://doi.org/10.3233/ch-141908.

96. Cantaluppi V, Gatti S, Medica D, Figliolini F, Bruno S, Deregibus MC, Sordi A, Biancone L, Tetta C, Camussi G. Microvesicles derived from endothelial progenitor cells protect the kidney from ischemia-reperfusion injury by microRNA-dependent reprogramming of resident renal cells. Kidney Int. 2012; 82:412-27. https://doi.org/10.1038/ ki.2012.105.

97. Wang Y, Lu X, He J, Zhao W. Influence of erythropoietin on microvesicles derived from mesenchymal stem cells protecting renal function of chronic kidney disease. Stem
Cell Res Ther. 2015; 6:100. https://doi.org/10.1186/ s13287-015-0095-0.

98. Erdbrugger U, Lannigan J. Analytical challenges of extracellular vesicle detection: A comparison of different techniques. Cytometry A. 2016; 89:123-34. https://doi. org/10.1002/cyto.a.22795.

99. Shah MD, Bergeron AL, Dong JF, Lopez JA. Flow cytometric measurement of microparticles: pitfalls and protocol modifications. Platelets. 2008; 19:365-72. https:// doi.org/10.1080/09537100802054107.

100. Gawaz M, Bogner C. Changes in platelet membrane glycoproteins and platelet-leukocyte interaction during hemodialysis. Clin Investig. 1994; 72:424-9.

101. Sanchez PG, Mackowick KM, Kon ZN. Current state of ex-vivo lung perfusion. Curr Opin Organ Transplant. 2016; 21:258-66. https://doi.org/10.1097/ mot.0000000000000310.

102. Cypel M, Keshavjee S. Extracorporeal lung perfusion (ex-vivo lung perfusion). Curr Opin Organ Transplant. 2016; 21:329-35. https://doi.org/10.1097/ mot.0000000000000320.

103. Macdonald PS, Chew HC, Connellan M, Dhital K. Extracorporeal heart perfusion before heart transplantation: the heart in a box. Curr Opin Organ Transplant. 2016; 21:336-42. https://doi.org/10.1097/mot.0000000000000309.

104. Yong C, Hosgood SA, Nicholson ML. Ex-vivo normothermic perfusion in renal transplantation: past, present and future. Curr Opin Organ Transplant. 2016; 21:301-7. https://doi.org/10.1097/mot.0000000000000316.

105. Okochi S, Cheung EW, Barton S, Zenilman A, Shakoor A, Street C, Streltsova S, Chan C, Brewer MP, Middlesworth W. An Analysis of Risk Factors for Hemolysis in Children on Extracorporeal Membrane Oxygenation. Pediatr Crit Care Med. 2018; 19:1059-1066 https://doi.org/10.1097/ pcc.0000000000001699.

106. Hennessy VL Jr, Hicks RE, Niewiarowski S, Edmunds LH Jr, Colman RW. Function of human platelets during extracorporeal circulation. Am J Physiol. 1977; 232: H622-8.

107. Boyd AD, Engelman RM, Beaudet RL, Lackner H. Disseminated intravascular coagulation following extracorporeal circulation. J Thorac Cardiovasc Surg. 1972; 64:685-93.

108. Kraft F, Schmidt C, Van Aken H, Zarbock A. Inflammatory response and extracorporeal circulation. Best Pract Res Clin Anaesthesiol. 2015; 29:113-23. https://doi.org/10.1016/j. bpa.2015.03.001.

109. Shaver CM, Upchurch CP, Janz DR, Grove BS, Putz ND, Wickersham NE, Dikalov SI, Ware LB, Bastarache JA. Cell-free hemoglobin: a novel mediator of acute lung injury. Am J Physiol Lung Cell Mol Physiol. 2016; 310:L532-41. https://doi.org/10.1152/ajplung.00155.2015.

110. Ray R, Kale PP, Ha R, Banerjee D. Treatment of left ventricular assist device-associated arteriovenous 
malformations with thalidomide. ASAIO J. 2014; 60:482-3. https://doi.org/10.1097/mat.0000000000000087.

111. Cheng C, van Haperen R, de Waard M, van Damme LC, Tempel D, Hanemaaijer L, van Cappellen GW, Bos J, Slager CJ, Duncker DJ, van der Steen AF, de Crom R, Krams R. Shear stress affects the intracellular distribution of eNOS: direct demonstration by a novel in vivo technique. Blood. 2005; 106:3691-8. https://doi.org/10.1182/ blood-2005-06-2326.

112. Silber HA, Bluemke DA, Ouyang P, Du YP, Post WS, Lima JA. The relationship between vascular wall shear stress and flow-mediated dilation: endothelial function assessed by phase-contrast magnetic resonance angiography. J Am Coll Cardiol. 2001; 38:1859-65.

113. Albalate M, Perez-Garcia R, de Sequera P, Corchete E, Alcazar R, Ortega M, Puerta M. Is it useful to increase dialysate flow rate to improve the delivered $\mathrm{Kt}$ ? BMC Nephrol. 2015; 16:20. https://doi.org/10.1186/ s12882-015-0013-9.

114. Ciandrini A, Lodi CA, Galato R, Miscia MC, Fattori MS, Cavalcanti S. A method for monitoring vascular access function during hemodialysis. Kidney Int. 2009; 75:550-7. https://doi.org/10.1038/ki.2008.581.

115. Mulhollandab JW, Shelton JC, Luo XY. Blood flow and damage by the roller pumps during cardiopulmonary bypass. J Fluids Struct. 2005; 20:129-40.

116. Wendler T, Schilling R, Lennertz A, Sodemann K, Kleophas W, Messner H, Riechers G, Wagner J, Keller C, Bosch T. Efficacy and safety of DALI LDL-apheresis at high blood flow rates: a prospective multicenter study. J Clin Apher. 2003; 18:157-66. https://doi.org/10.1002/jca.10071.

117. Slaughter MS, Sobieski MA 2nd, Tamez D, Horrell T, Graham J, Pappas PS, Tatooles AJ, LaRose J. HeartWare miniature axial-flow ventricular assist device: design and initial feasibility test. Tex Heart Inst J. 2009; 36:12-6.

118. Wernicke JT, Meier D, Mizuguchi K, Damm G, Aber G, Benkowski R, Nose Y, Noon GP, DeBakey ME. A fluid dynamic analysis using flow visualization of the Baylor/ NASA implantable axial flow blood pump for design improvement. Artif Organs. 1995; 19:161-77.

119. Mallick A, Elliot S, McKinlay J, Bodenham A. Extracorporeal carbon dioxide removal using the Novalung in a patient with intracranial bleeding. Anaesthesia. 2007; 62:72-4. https://doi.org/10.1111/j.1365-2044.2006.04863.X.

120. Gu K, Zhang Y, Gao B, Chang Y, Zeng Y. Hemodynamic Differences Between Central ECMO and Peripheral ECMO: A Primary CFD Study. Med Sci Monit. 2016; 22:717-26.

121. Vermeulen Windsant IC, de Wit NC, Sertorio JT, van Bijnen AA, Ganushchak YM, Heijmans JH, Tanus-Santos JE, Jacobs MJ, Maessen JG, Buurman WA. Hemolysis during cardiac surgery is associated with increased intravascular nitric oxide consumption and perioperative kidney and intestinal tissue damage. Front Physiol. 2014; 5:340. https:// doi.org/10.3389/fphys.2014.00340.
122. Bottrell S, Bennett M, Augustin S, Thuys C, Schultz B, Horton A, Horton S. A comparison study of haemolysis production in three contemporary centrifugal pumps. Perfusion. 2014; 29:411-6. https://doi. org/10.1177/0267659113509000.

123. Rodrigo R, Libuy M, Feliu F, Hasson D. Oxidative stressrelated biomarkers in essential hypertension and ischemiareperfusion myocardial damage. Dis Markers. 2013; 35:773-90. https://doi.org/10.1155/2013/974358.

124. Mohandas R, Segal MS. Endothelial progenitor cells and endothelial vesicles - what is the significance for patients with chronic kidney disease? Blood Purif. 2010; 29:158-62. https://doi.org/10.1159/000245643.

125. Pieri M, Greco T, De Bonis M, Maj G, Fumagalli L, Zangrillo A, Pappalardo F. Diagnosis of infection in patients undergoing extracorporeal membrane oxygenation: a casecontrol study. J Thorac Cardiovasc Surg. 2012; 143:1411-6. https://doi.org/10.1016/j.jtcvs.2012.01.005.

126. Roth C, Schrutka L, Binder C, Kriechbaumer L, Heinz G, Lang IM, Maurer G, Koinig H, Steinlechner B, Niessner A, Distelmaier K, Goliasch G. Liver function predicts survival in patients undergoing extracorporeal membrane oxygenation following cardiovascular surgery. Crit Care. 2016; 20:57. https://doi.org/10.1186/s13054-016-1242-4.

127. Distelmaier K, Roth C, Binder C, Schrutka L, Schreiber C, Hoffelner F, Heinz G, Lang IM, Maurer G, Koinig H, Steinlechner B, Niessner A, Goliasch G. Urinary Output Predicts Survival in Patients Undergoing Extracorporeal Membrane Oxygenation Following Cardiovascular Surgery. Crit Care Med. 2016; 44:531-8. https://doi.org/10.1097/ ccm.0000000000001421.

128. Dewanjee MK, Wu SM, Kapadvanjwala M, De D, Dewanjee S, Novak S, Hsu LC, Perryman RA, Serafini AN, Sfakianakis GN, Duncan RC, Dietrich WD, Horton AF. Reduction of Platelet Thrombi and Emboli by L-Arginine during Cardiopulmonary Bypass in a Pig Model. J Thromb Thrombolysis. 1996; 3:343-60.

129. van den Goor JM, Nieuwland R, Rutten PM, Tijssen JG, Hau C, Sturk A, Eijsman L, de Mol BA. Retransfusion of pericardial blood does not trigger systemic coagulation during cardiopulmonary bypass. Eur J Cardiothorac Surg. 2007; 31:1029-36. https://doi.org/10.1016/j. ejcts.2007.02.016.

130. Nomura S, Inami N, Kanazawa S, Iwasaka T, Fukuhara S. Elevation of platelet activation markers and chemokines during peripheral blood stem cell harvest with G-CSF. Stem Cells. 2004; 22:696-703. https://doi.org/10.1634/ stemcells.22-5-696.

131. Wun T, Paglieroni T, Holland P. Prolonged circulation of activated platelets following plasmapheresis. J Clin Apher. 1994; 9:10-6. 